This is a self-archived version of an original article. This version may differ from the original in pagination and typographic details.

Author(s): Iwaniec, Tadeusz; Onninen, Jani

Title: Radó-Kneser-Choquet Theorem for simply connected domains (p-harmonic setting)

Year: 2019

Version: Accepted version (Final draft)

Copyright: @ 2018 American Mathematical Society

Rights: $C C B Y-N C-N D 4.0$

Rights url: https://creativecommons.org/licenses/by-nc-nd/4.0/

Please cite the original version:

Iwaniec, T., \& Onninen, J. (2019). Radó-Kneser-Choquet Theorem for simply connected domains (p-harmonic setting). Transactions of the American Mathematical Society, 371(4), 2307-2341.

https://doi.org/10.1090/tran/7348 


\title{
RADÓ-KNESER-CHOQUET THEOREM FOR SIMPLY CONNECTED DOMAINS (P-HARMONIC SETTING)
}

\author{
TADEUSZ IWANIEC AND JANI ONNINEN
}

\begin{abstract}
A remarkable result known as Radó-Kneser-Choquet theorem asserts that the harmonic extension of a homeomorphism of the boundary of a Jordan domain $\Omega \subset \mathbb{R}^{2}$ onto the boundary of a convex domain $\mathcal{Q} \subset \mathbb{R}^{2}$ takes $\Omega$ diffeomorphically onto $\mathcal{Q}$. Numerous extensions of this result for linear and nonlinear elliptic PDEs are known, but only when $\Omega$ is a Jordan domain or, if not, under additional assumptions on the boundary map. On the other hand, the newly developed theory of Sobolev mappings between Euclidean domains and Riemannian manifolds demands to extend this theorem to the setting on simply connected domains. This is the primary goal of our article. The class of the $p$-harmonic equations is wide enough to satisfy those demands. Thus we confine ourselves to considering the $p$-harmonic mappings.

The situation is quite different than that of Jordan domains. One must circumvent the inherent topological difficulties arising near the boundary.

Our main THEOREM 4 is the key to establishing approximation of monotone Sobolev mappings with diffeomorphisms. This, in turn, leads to the existence of energy-minimal deformations in the theory of Nonlinear Elasticity. Hence the usefulness of THEOREM 4. We do not enter these applications here, but refer the reader to Section 1.2, for comments. .
\end{abstract}

\section{COnTEnts}

1. Introduction 2

1.1. $p$-harmonic mappings $\quad 2$

1.2. Historical account and related comments 4

1.3. General plan 6

2010 Mathematics Subject Classification. Primary 30E10; Secondary 46E35, $58 \mathrm{E} 20$.

Key words and phrases. Harmonic mappings, p-harmonic equation, monotone mappings.

T. Iwaniec was supported by the NSF grant DMS-1301558. J. Onninen was supported by the NSF grant DMS-1700274. 
2. Complex gradient of a $p$-harmonic function 8

2.1. Stoilow Factorization 9

2.2. Critical Points 9

3. The $(p, q)$-holomorphic pair, $\frac{1}{p}+\frac{1}{q}=1 \quad 10$

4. Increment of arguments 13

4.1. Tangent Vector Field 14

4.2. Curved Polygons 16

5. The curved $4 m$-polygon around the critical point 18

6. The relationship $m=n+1 \quad 21$

7. The $p$-harmonic Dendrite 23

8. Generalized Crosscut of a Simply-Connected Domain 26

8.1. A refinement of Theorem 20 and Lemma $23 \quad 30$

9. No critical points of $U$ and $V$

9.1. Jacobian determinant 34

10. An Elliptic Equation for $W=\alpha U+\beta V$

11. The $\mathcal{A}$-Harmonic Dendrite of $W=\alpha U+\beta V \quad 39$

12. Global Injectivity 41

Acknowledgement $\quad 43$

References $\quad 43$

\section{INTRODUCTION}

The basis for the discussion of our main results is the theorem of Radó-Kneser-Choquet. We shall abbreviate it to RKC-theorem.

Theorem 1 (Radó-Kneser-Choquet). Let $h=u+i v: \partial \Omega \stackrel{\text { onto }}{\longrightarrow} \partial \mathcal{Q}$ be a homeomorphism of the boundary of a (bounded) Jordan domain $\Omega \subset \mathbb{R}^{2}$ onto the boundary of a convex domain $\mathcal{Q} \subset \mathbb{R}^{2}$. Then its continuous harmonic extension $H=U+i V$ is a $\mathscr{C}^{\infty}$-diffeomorphism of $\Omega$ onto $\mathcal{Q}$.

A brief historical account and extensions of this theorem in different directions are given in Subsection 1.2. It is Kneser's original proof [34] that underlies the basic ideas in the present paper. Thus in Subsection 1.3 we sketch his proof. This will help us organize and confer about our own ingredients.

1.1. $p$-harmonic mappings. We are concerned with mappings $H=$ $U+i V$ defined in a bounded simply connected domain $\Omega \subset \mathbb{R}^{2} \simeq \mathbb{C}$, 
whose coordinate functions $U$ and $V$ satisfy the $p$-harmonic equation,

$$
\left\{\begin{array}{l}
\operatorname{div}|\nabla U|^{p-2} \nabla U=0 \\
\operatorname{div}|\nabla V|^{p-2} \nabla V=0
\end{array} \quad U, V \in \mathscr{W}_{\operatorname{loc}}^{1, p}(\Omega), \quad 1<p<\infty\right.
$$

A marked difference between (1.1) and the commonly studied coupled $p$-harmonic system $\operatorname{div}|\mathcal{D} H|^{p-2} \mathcal{D} H=0$ should be noted. The latter has been considered in [24] with the purpose of generalizing the RKC-theorem for coupled systems. The task was accomplished for smooth domains. Solving it in a general setting seems to be complicated because of shortage of estimates up to the boundary. One major advantage of using (1.1) lies in the existence and uniqueness of the solution to the Dirichlet problem in simply connected domains. Let us briefly outline the key points of the arguments for such a general Dirichlet problem, and refer to [21] and [40] for a thorough treatment. By virtue of the famous Wiener's criterion the existence and uniqueness of the solution holds whenever the complement $\mathbb{R}^{2} \backslash \Omega$ is $p$-thick at every boundary point, see [40, Corollary 6.22] and [40, (2.22)] for a formulation of Wiener's criterion. Simply connected domains indeed satisfy this criterion, a fact not difficult to verify though not explicitly stated in the literature.

Proposition 2. Let $\Omega$ be a bounded simply connected domain in $\mathbb{R}^{2}$. Then every continuous function $u: \partial \Omega \rightarrow \mathbb{R}$ admits a unique continuous extension, denoted by $U \in \mathscr{C}(\bar{\Omega}) \cap \mathscr{W}_{\mathrm{loc}}^{1, p}(\Omega)$, which is $p$-harmonic in $\Omega$.

We refer to $U: \Omega \rightarrow \mathbb{R}$ as the $p$-harmonic extension of $u$.

First observe that when $\Omega$ is not a Jordan domain, one cannot speak of a homeomorphism from $\partial \Omega \stackrel{\text { onto }}{\longrightarrow} \partial \mathcal{Q}$. A relevant notion in this context is that of monotone boundary map. C. B. Morrey [45] was the first to propose the concept of a monotone map between general topological spaces. Let us phrase his definition in the form suitable for our purposes.

Definition 3. A continuous map $h: \mathbb{X} \stackrel{\text { onto }}{\longrightarrow} \mathbb{Y}$ between compact metric spaces is monotone if the preimage $h^{-1}\left(y_{\circ}\right)$ of every point $y_{\circ} \in$ $\mathbb{Y}$ is a continuum (connected and compact) in $\mathbb{X}$. This yields, as shown by Whyburn, that the preimage of every connected subset of $\mathbb{Y}$ is connected in $\mathbb{X}$, see $[42$, page 2$]$.

Description of a monotone map $h=u+i v: \partial \Omega \stackrel{\text { onto }}{\longrightarrow} \partial \mathcal{Q}$ between Jordan curves is rather straightforward. Namely; the preimages of points in $\partial \mathcal{Q}$, except for a countable set of them, are single points 
in $\partial \Omega$ while preimages of the remaining exceptional points are compact Jordan arcs in $\partial \Omega$. If $\Omega$ is only simply connected, the lack of such description of the monotone boundary map is the main source of difficulties in this paper. Nonetheless, we succeeded in proving the following theorem, which is the main result of the present paper.

THEOREM 4. Consider a bounded simply connected domain $\Omega \subset \mathbb{R}^{2}$ and a bounded convex domain $\mathcal{Q} \subset \mathbb{R}^{2}$. Let $h: \partial \Omega \stackrel{\text { onto }}{\longrightarrow} \partial \mathcal{Q}$ be a continuous monotone mapping and $H: \Omega \rightarrow \mathbb{R}^{2}$ denote its p-harmonic extension, $1<p<\infty$. Then $H$ is a $\mathscr{C}^{\infty}$-diffeomorphism of $\Omega$ onto $\mathcal{Q}$.

This theorem, since it deals with general simply connected domains, is new even in the case of harmonic mappings $(p=2)$.

With the aid of a Riemann conformal transformation of the target domain the following topological fact is immediate.

Corollary 5. Every monotone map $h: \partial \Omega \stackrel{\text { onto }}{\longrightarrow} \partial \mathcal{U}$ of the boundary of a simply connected domain onto the boundary of a Jordan domain admits a continuous extension to $\bar{\Omega}$ which takes $\Omega$ homeomorphically onto $\mathcal{U}$.

Remark 6. One could simplify some of the topological arguments in this paper if we knew in advance that the monotone boundary map $h$ : $\partial \Omega \stackrel{\text { onto }}{\longrightarrow} \partial \mathcal{Q}$ indeed admits a homeomorphic extension $\mathcal{H}: \Omega \stackrel{\text { onto }}{\longrightarrow} \mathcal{Q}$, see [27]. In the light of this remark a question arises whether Corollary 5 remains valid if $\mathcal{U}$ is only simply connected? The answer is not known to us.

1.2. Historical account and related comments. Theorem 1 was conjectured in 1926 by Radó [48] and proved the same year by Kneser [34], see also [22, p. 78-80] for a nice presentation. Choquet [16], apparently unaware of Kneser's work, gave his own proof. Regarding more recent approach, let us note that the univalence property of $H: \Omega \stackrel{\text { onto }}{\longrightarrow} \mathcal{Q}$ is unaffected if one performs an isotopy of the boundary homeomorphism. This is due to the minimum principle of the Jacobian determinant. In [29] we constructed an isotopy that connects $h: \partial \Omega \stackrel{\text { onto }}{\longrightarrow} \partial \mathcal{Q}$ with a boundary homeomorphism $h_{\circ}: \partial \Omega \stackrel{\text { onto }}{\longrightarrow} \partial \mathcal{Q}$ whose harmonic extension is holomorphic in $\Omega$. This latter becomes a conformal homeomorphism regardless of convexity of $\partial \mathcal{Q}$. This new idea has led us to yet another proof of RKC-theorem. It was also explored in [24] for the nonlinear coupled isotropic $p$-harmonic systems. The interested reader is referred to [32] and [49] for further development in the manifold setting. A full treatment of the RKC-theorem for linear second-order 
elliptic systems can be found in $[3,4,5,11]$. We aslo refer to $[18,38]$ for information about harmonic transformations of multiply connected domains.

Actually, the inspiration for THEOREM 4 was the paper of G. Alessandrini and M. Sigalotti [7] on the extension of RKC-theorem to the anisotropic $p$-Laplace type systems. This paper provided us with the essential tool for establishing strong approximation of Sobolev homeomorphisms with diffeomorphism, see [25]. The novelty of the paper by Alessandrini and Sigalottii lies in finding a second order elliptic equation for a linear combination $\alpha U+\beta V$ of the $p$-harmonic functions $U$ and $V$. We exploit this idea in Section 10.

For more related results and comments about harmonic mappings, we refer to Duren's book [17] and to the survey article by Bshouty and Hengartner [15].

1.2.1. Further comments. RKC-theorem is extremely sensitive to slight changes in its hypotheses, see the first two itemized comments.

- If the target (a Jordan domain $\mathcal{Q} \subset \mathbb{R}^{2}$ ) fails to be convex, one can always find a homeomorphism $h: \partial \Omega \stackrel{\text { onto }}{\longrightarrow} \partial \mathcal{Q}$ whose harmonic extension fails to be injective. This was already observed by Choquet [16]. Nevertheless, the convexity assumption on $\mathcal{Q}$ is redundant if the harmonic extension happens to take $\Omega$ onto $\mathcal{Q}$. For this and for further results about non-convex domains we refer to $[5,33]$.

- Let us stress that all the existing extensions of RKC-theorem to linear and nonlinear PDEs, except for [12, 24], deal essentially with the systems in which both $U$ and $V$ satisfy the same equation. Notably, any small perturbation of only one of the equations in the system (1.1) results in failure of the RKC-theorem [6]. Therefore, possible changes in RKC-theorem should be made simultaneously in both equations. But how?

- Except for the harmonic case of $p=2$, the system (1.1) is not invariant under a rotation of the domain $\Omega$, neither it is invariant under a rotation of the target $\mathcal{Q}$. Different choices of the coordinates through rotations result in new systems of PDEs. In general, These new systems become coupled systems. Nonetheless, the RKC-theorem still applies. One must admit, therefore, that the unisotropic $p$-harmonic mappings (as defined by 1.1) are not geometric objects. Also from a physical perspectives the mapping $H=U+i V$ cannot be interpreted deformation of an elastic body. 
- From this point of view, the general problem of extending RKCtheorem to coupled systems of PDEs which are coordinate free , partially solved in [24] for isotropic $p$ harmonic system with "nice" boundary data is important. The problem remains open.

- The RKC-theorem for harmonic mappings fails in higher dimensions. Indeed [36], Laugesen constructed a self-homeomorphism of the sphere in $\mathbb{R}^{n}, n \geqslant 3$. whose harmonic extension to the ball is not injective. In a related construction for $\mathbb{R}^{3}$, Melas [43] showed that the harmonic extension of a homeomorphism of sphere need not be a diffeomorphism. However, Melas' construction is impossible under an additional hypothesis on the harmonic mapping. For example, if a homeomorphism (in 3D) is the gradient of a real-valued harmonic function, it is automatically a diffeomorphism $[19,37]$.

- In this latter case, what are the variational-energy-problems for 3D-deformations whose minima are gradients of harmonic functions?

- Are the total energy-minimal maps, introduced in [28], diffeomorphisms if the boundary data is a homeomorphism?

Even in the planar case very little is known about this question. The existing methods fall short of providing sufficient (Lipschitz) regularity of the minimal maps. To illustrate, examine the simplest total energy integral,

$$
\mathscr{E}[H] \stackrel{\text { def }}{=} \int_{\Omega}\left(|\mathcal{D} H(x)|^{2}+\frac{|\mathcal{D} H(x)|^{2}}{\operatorname{det} \mathcal{D} H(x)}\right) \mathrm{d} x
$$

1.3. General plan. All the significant extensions and refinements of RKC-theorem can be derived from the same scheme of ideas found in [34]. In brief outline, here are the key steps which give guidance to our proofs as well. Thus let us look, temporarily, at the harmonic extension $H=U+i V: \Omega \stackrel{\text { onto }}{\longrightarrow} \mathcal{Q}$, see Figure 1 .

(A) First we aim to establish local injectivity of $H$ by showing that the Jacobian determinant $J_{H}(z)=U_{x} V_{y}-U_{y} V_{x}$ does not vanish in $\Omega$.

(B) Suppose that, on the contrary, $J_{H}\left(z_{\circ}\right)=0$. This means that there is a nontrivial linear combination $W \stackrel{\text { def }}{=} \alpha U+\beta V$ (which is also a nonconstant harmonic function) whose gradient vanishes at $z_{0}$.

(C) The complex gradient $\frac{\partial W}{\partial z} \stackrel{\text { def }}{=} \frac{1}{2}\left(W_{x}-i W_{y}\right)$, being a nonconstant holomorphic function, admits only isolated zeros. 
(D) Striving for a contradiction, we look at the level set

$$
\mathfrak{L}=\left\{z \in \Omega ; W(z)=W\left(z_{\circ}\right)\right\}
$$

(E) The local structure of $\mathfrak{L}$ near $z_{\circ}$ is established by considering a holomorphic function $F \stackrel{\text { def }}{=} W+i \widetilde{W}$, where $\widetilde{W}$ stands for a harmonic conjugate of $W$. The derivative of $F$ vanishes at $z_{\circ}$. In this was complex function theory comes into play. It tells us that from $z_{0}$ there emanate $2 n+2$ Jordan arcs, where $n$ is the order of zero of $F^{\prime}$ at $z_{0}$. In particular, $2 n+2 \geqslant 4$.

(F) Each of the above arcs extends along $\mathfrak{L}$ and terminates at exactly one point on $\partial \Omega$. Call it the endpoint of the arc. No two of the extended arcs can meet in $\Omega$. We have $2 n+2$ endpoints (possibly with repetition) in $\partial \Omega$.

(G) We look at the homeomorphism $H=U+i V: \partial \Omega \stackrel{\text { onto }}{\longrightarrow} \partial \mathcal{Q}$ and the straight line $\left\{(U, V) \in \mathbb{R}^{2}: \alpha U+\beta V \equiv\right.$ constant $=$ $\left.W\left(z_{\circ}\right)\right\}$ in the $(U, V)$-plane. Typically, this line intersects a convex curve like $\partial \mathcal{Q}$ at two distinct points, sometimes at one point or along a line segment which happens to be a part of $\partial \mathcal{Q}$. In either case, we may select two extended arcs emanating from $z_{\circ}$ which terminate at the same point on $\partial \Omega$, or their endpoints are connected by a Jordan arc in $\partial \Omega$ along which $W=\alpha U+\beta V \equiv$ constant $=W\left(z_{\circ}\right)$. In this way we obtain a simple closed Jordan curve along which $W$ is constant (in case of a simply connected domain and the monotone boundary map a similar construction will be more complicated).

(H) The bounded complementary domain of the above constructed Jordan curve must lie in $\Omega$. It then follows that $W$ is constant in this complementary domain. By virtue of the unique continuation property, $W$ is constant in the entire $\Omega$. This is a clear contradiction of the fact that $H=U+i V: \partial \Omega \stackrel{\text { onto }}{\longrightarrow} \partial \mathcal{Q}$ is a homeomorphism.

(I) Therefore, $H$ is a local homeomorphism in $\Omega$; actually a local diffeomorphism. Finally, global injectivity of $H$ follows by a general topological reasoning. We shall appeal to the original work of Banach and Mazur [10].

Our proof of THEOREM 4 is organized along similar lines. However, all of these lines require their own further considerations and new ingredients. Through Sections 2-7, we present the detailed proofs of some general facts together with comments which might be of independent 


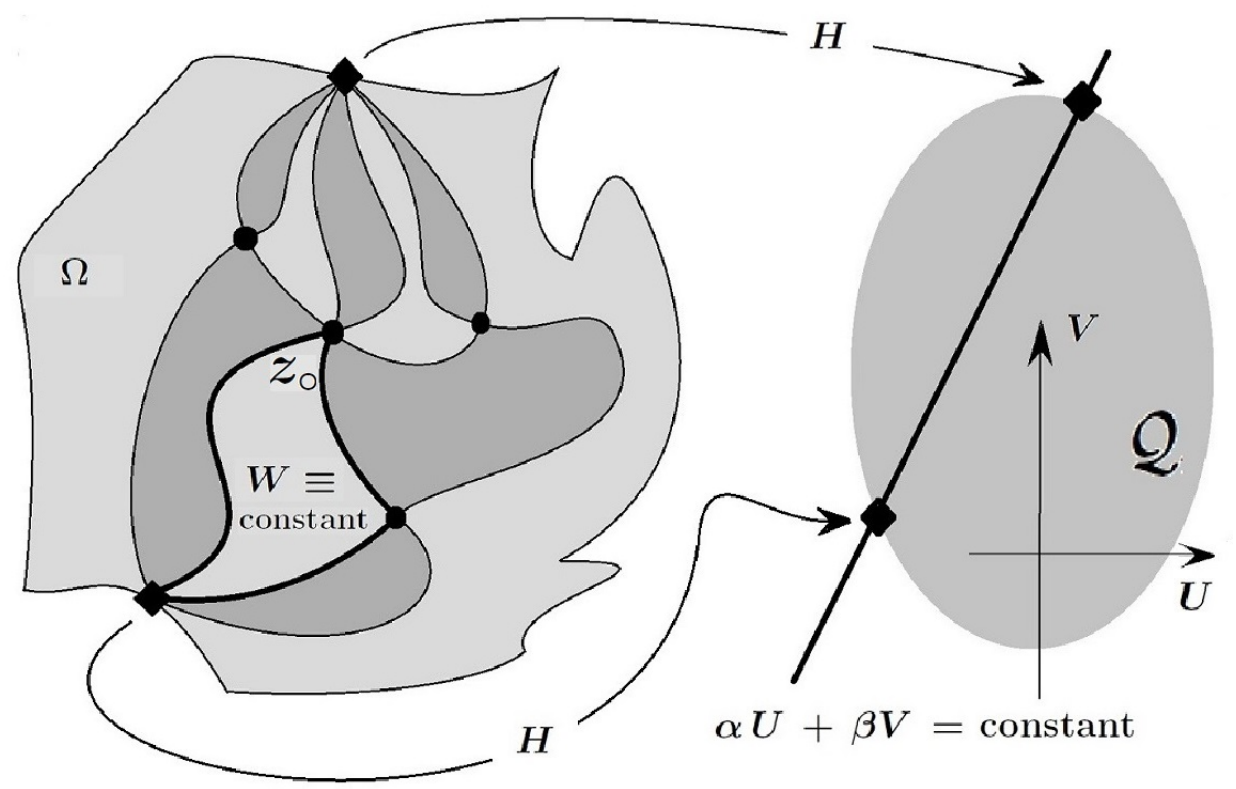

Figure 1. Kneser's proof of RKC-theorem in one drawing.

interest. Certainly, new topological ideas are necessary when studying the behavior of the level curves near the boundary. We aim to present these ideas and arguments in a perfectly rigorous manner, either by providing complete proofs or referring to equally rigorous papers. This lengthens the paper.

\section{Complex Gradient of A $p$-HARMOniC FUnCTiON}

Whenever it is convenient, we shall freely identify the vector space $\mathbb{R}^{2}$ with the complex plane $\mathbb{C}$. We will use the symbols $\mathbb{R}_{\circ}^{2}=\mathbb{R}^{2} \backslash\{0\}$ and $\mathbb{C}_{\circ}=\mathbb{C} \backslash\{0\}$ of the punctured plane.

Consider a nonconstant solution of the $p$-harmonic equation in a domain $\Omega \subset \mathbb{R}^{2} \simeq \mathbb{C}$,

$$
\operatorname{div}|\nabla u|^{p-2} \nabla u=0, \quad u \in \mathscr{W}_{\mathrm{loc}}^{1, p}(\Omega), 1<p<\infty
$$

Its complex gradient $f(z) \stackrel{\text { def }}{=} \frac{1}{2}\left(u_{x}-i u_{y}\right)=u_{z}$ turns out to be a $\mathscr{W}_{\text {loc }}^{1,2}(\Omega)$-solution of a quasi-linear uniformly elliptic equation [13] (for $p \geqslant 2$ ) and extended to $1<p \leqslant 2$ in $[41,26]$.

$$
\frac{\partial f}{\partial \bar{z}}=\frac{2-p}{2 p}\left\{\frac{\bar{f}}{f} \frac{\partial f}{\partial z}+\frac{f}{\bar{f}} \frac{\overline{\partial f}}{\partial z}\right\}, z=x+i y
$$


In particular, $f$ satisfies a Beltrami type equation

$$
\frac{\partial f}{\partial \bar{z}}=\mu(z) \frac{\partial f}{\partial z}, \quad \text { where }|\mu(z)| \leqslant k=\left|1-\frac{2}{p}\right|<1
$$

Recall that the term $K$-quasiregular mapping refers to an $f \in \mathscr{W}_{\text {loc }}^{1,2}(\Omega, \mathbb{C})$ whose distortion function $K_{f}(z) \stackrel{\text { def }}{=} \frac{|D f(z)|^{2}}{J_{f}(z)}$ is bounded by $K, 1 \leqslant$ $K<\infty$. We have,

$$
K_{f}(z)=\frac{\left(\left|f_{z}\right|+\left|f_{\bar{z}}\right|\right)^{2}}{\left|f_{z}\right|^{2}-\left|f_{\bar{z}}\right|^{2}}=\frac{1+|\mu(z)|}{1-|\mu(z)|} \leqslant \max \left\{p-1, \frac{1}{p-1}\right\}
$$

Thus the complex gradient $f=u_{z}$ is a $K$-quasiregular mapping with $K=\max \left\{p-1, \frac{1}{p-1}\right\}$. It is known [46] that every $K$-quasiregular mapping is locally Hölder continuous of exponent $\alpha=\frac{1}{K}$. In particular,

$$
u \in \mathscr{C}_{\mathrm{loc}}^{1, \alpha}(\Omega), \text { with } \alpha=\min \left\{p-1, \frac{1}{p-1}\right\}
$$

The interested reader is referred to [26] for an optimal Hölder exponent and other sharp regularity results.

2.1. Stoilow Factorization. The principal feature of the solutions to the Beltrami equation (2.2) is the following Stoilow factorization, first established by C.B. Morrey [46].

$$
f(z)=\mathcal{H}(\phi(z)),
$$

where $\phi: \Omega \stackrel{\text { onto }}{\longrightarrow} \phi(\Omega)$ is a quasiconformal homeomorphism, which also solves the Beltrami equation (2.2), and $\mathcal{H}: \phi(\Omega) \rightarrow \mathbb{C}$ is a holomorphic function.

2.2. Critical Points. As a consequence of Stoilow's factorization we see that $f$ has isolated zeros. These are the critical points of $u$. Let us introduce the domain $\Omega_{\circ}$ obtained from $\Omega$ by removing zeros of $f$. It then follows from Equation (2.1) that $f \in \mathscr{C}^{\infty}\left(\Omega_{\circ}\right)$. A detailed proof can be found in [9, Theorem 15.7.1, p. 400].

We are going to examine the local structure of the level curves of $u$ near its critical point. For this we may (and do) restrict ourselves to a small neighborhood of the isolated critical point. Let us assume that the origin $0 \in \mathbb{C}$ is a critical point and $\Omega$ is a small neighborhood of 0 which contains no other critical points. Thus the holomorphic function $\mathcal{H}$ in $(2.5)$ takes the form $\mathcal{H}(\zeta)=[\Phi(\zeta)]^{n}, \Phi^{\prime}(0) \neq 0$, where $n \geqslant 1$ is the order of zero of $\mathcal{H}$, also referred to as the order of the critical 
point of $u$. In fact $\Phi=\sqrt[n]{\mathcal{H}}$ is a continuous branch of the $n$-th root of $\mathcal{H}$.

We may, if necessary, further restrict $f$ to somewhat smaller neighborhood of 0 , again denoted by $\Omega$, so that the mapping $\chi(z) \stackrel{\text { def }}{=}$ $\Phi(\phi(z))$ is a $K$-quasiconformal homeomorphism in $\Omega$.

Proposition 7. We have a Stoilow factorization

$$
f(z)=[\chi(z)]^{n}, \quad \chi(0)=0
$$

where $\chi: \Omega \stackrel{\text { onto }}{\longrightarrow} \chi(\Omega)$ is a quasiconformal homeomorphism. Actually $\chi$ is a $\mathscr{C}^{\infty}$-diffeomorphism in $\Omega_{\circ}=\Omega \backslash\{0\}$.

Proof. To see that $\chi$ is $\mathscr{C}^{\infty}$-diffeomorphism in $\Omega_{\circ}$, we substitute $f$ from (2.6) into (2.1) to obtain a quasilinear elliptic equation for $\chi$,

$$
\frac{\partial \chi}{\partial \bar{z}}=\frac{2-p}{2 p}\left\{\frac{\bar{\chi}^{n}}{\chi^{n}} \frac{\partial \chi}{\partial z}+\frac{\chi^{n}}{\bar{\chi}^{n}} \frac{\overline{\partial \chi}}{\partial z}\right\}
$$

The equation for the inverse map $\digamma=\digamma(\xi)=\chi^{-1}(\xi)$ becomes linear and uniformly elliptic.

$$
\frac{\partial \digamma}{\partial \bar{\xi}}=\frac{p-2}{2 p}\left\{\frac{\bar{\xi}^{n}}{\xi^{n}} \frac{\overline{\partial \digamma}}{\partial \xi}+\frac{\xi^{n}}{\bar{\xi}^{n}} \frac{\partial \digamma}{\partial \xi}\right\}
$$

We again appeal to the regularity result in [9, Theorem 15.7.1, p. 400] to infer that both $\chi$ and $\chi^{-1}$ are $\mathscr{C}^{\infty}$ smooth outside the origin.

\section{The $(p, q)$-HOLOMORPHIC PAIR, $\frac{1}{p}+\frac{1}{q}=1$}

To every $p$ harmonic function $u$, defined in a simply connected domain $\Omega$ there corresponds a $q$-harmonic conjugate function $v$ (stream function). The pair $(u, v)$ satisfies a first order system of PDEs,

$$
\left\{\begin{array}{l}
|\nabla u|^{p-2} u_{x}=v_{y} \\
|\nabla u|^{p-2} u_{y}=-v_{x}
\end{array} \text {, thus } v \in \mathscr{W}_{\mathrm{loc}}^{1, q}(\Omega) \text { is } q-\right.\text { harmonic }
$$

An analogy with the Cauchy-Riemann equation motivates us calling the complex function $F \stackrel{\text { def }}{=} u+i v$ a $(p, q)$-holomorphic pair. The gradients $\nabla u$ and $\nabla v$ are orthogonal and vanish at the same set of points, which is the common set of critical points of $u$ and $v$. Thus, by (2.4) $F \in \mathscr{C}_{\text {loc }}^{1, \alpha}(\Omega)$. Outside the critical points $F$ is $\mathscr{C}^{\infty}$ smooth. Compute its complex derivatives 


$$
\begin{aligned}
2 F_{\bar{z}} & =F_{x}+i F_{y}=u_{x}+i v_{x}+i u_{y}-v_{y}=\left(1-|\nabla u|^{p-2}\right)\left(u_{x}+i u_{y}\right) \\
& =2\left(1-|f|^{p-2}\right) \bar{f}
\end{aligned}
$$

Similarly,

$$
\begin{aligned}
2 F_{z} & =F_{x}-i F_{y}=u_{x}+i v_{x}-i u_{y}+v_{y}=\left(1+|\nabla u|^{p-2}\right)\left(u_{x}-i u_{y}\right) \\
& =2\left(1+|f|^{p-2}\right) f
\end{aligned}
$$

we obtain a complex Beltrami equation in $\Omega$

$$
F_{\bar{z}}=\mu(z) F_{z}, \quad \text { with } \mu=\frac{\left(1-|f|^{p-2}\right) \bar{f}}{\left(1+|f|^{p-2}\right) f} \in \mathscr{C}^{\infty}\left(\Omega_{\circ}\right)
$$

Throughout, we confine ourselves to discussing $F$ in a small neighborhood of one (and only one) critical point, say at $0 \in \Omega$. Furthermore, it simplifies the writing and causes no loss of generality, to assume that such a neighborhood is the unit disk $\{z \in \mathbb{C}:|z|<1\}=\mathbb{D}$ (by rescaling if necessary).

Recall the distortion function

$$
K_{F}(z) \stackrel{\text { def }}{=} \frac{1+|\mu(z)|}{1-|\mu(z)|}=\max \left\{|f(z)|^{p-2},|f(z)|^{2-p}\right\}
$$

Equivalently,

$$
K_{F}(z)=\left\{\begin{array}{ll}
|f(z)|^{p-2}, & \text { if } 1<p \leqslant 2 \\
|f(z)|^{2-p}, & \text { if } 2 \leqslant p
\end{array}\right\}=|f(z)|^{-|p-2|}, \quad \text { for } z \neq 0
$$

Next we invoke Stoilow's factorization in (2.6), $f(z)=[\chi(z)]^{n}$, where $\chi$ is a $K$-quasiconformal homeomorphism with $K=\max \{p-$ $1,1 / p-1\}$. This yields an estimate $|\chi(z)| \geqslant c|z|^{K}$ near the origin. The interested reader is referred to [26] for sharp regularity results. Thus, $|f(z)| \geqslant c|z|^{n K}$ and, therefore,

$$
K_{F}(z) \leqslant C|z|^{-n K|p-2|}, \text { near } z=0
$$

We shall make a radial change of $z$-variable, in the interest to obtain a mapping whose distortion function is $\mathscr{L}^{1}$-integrable near the origin. To this effect consider a map defined in $\Omega$ by the rule

$$
\begin{gathered}
G(z) \stackrel{\text { def }}{=} F(\Psi(z)) \quad \text {, where } \\
\Psi(z)=|z|^{\varepsilon-1} z \quad, \quad 0<\varepsilon<\min \left\{1, \frac{2}{n K|p-2|}\right\}
\end{gathered}
$$


Clearly $G \not \equiv$ constant. Moreover, $G$ lies in the Sobolev space $\mathscr{W}^{1,2}(\mathbb{D})$. In fact we have explicit $\mathscr{W}^{1,2}$-estimates. To see these estimates first observe that the distortion function of $\Psi$ is constant; indeed, $K_{\Psi}(z) \stackrel{\text { def }}{=}$ $|D \Psi(z)|^{2} / J_{\Psi}(z) \equiv \frac{1}{\varepsilon}$. The chain rule yields, $D G(z)=D F(\Psi(z) \circ$ $D \Psi(z)$ and hence

$$
|D G(z)|^{2} \leqslant|D F(\Psi(z))|^{2} \cdot|D \Psi(z)|^{2} \leqslant \frac{1}{\varepsilon} \mid D F\left(\left.\Psi(z)\right|^{2} J_{\Psi}(z)\right.
$$

Upon integration over the unit disk (by a change of variables) we infer that

$$
\int_{\Omega}|D G(z)|^{2} \mathrm{~d} z \leqslant \frac{1}{\varepsilon} \int_{\Psi(\Omega)}|D F(w)|^{2} \mathrm{~d} w<\infty
$$

Furthermore, the distortion function of $G$ is $\mathscr{L}^{1}$-integrable, because

$$
K_{G}(z) \leqslant K_{F}(\Psi(z)) \cdot K_{\Psi}(z) \leqslant \frac{C}{\varepsilon}|\Psi(z)|^{-n K|p-2|}=\frac{C}{\varepsilon}|z|^{-\varepsilon n K|p-2|}
$$

We are now in a position to appeal to Stoilow's factorization of mappings with integrable distortion. It asserts that, see [30].

Proposition 8. Suppose a function $G \in \mathscr{W}^{1,2}(\mathbb{D})$, such as the one in (3.3), satisfies the distortion equation

$$
|D G(z)|^{2}=K_{G}(z) J_{G}(z), \text { where } K_{G} \in \mathscr{L}^{1}(\mathbb{D})
$$

Then $G(z)=\mathcal{H}(\Phi(z))$, where $\Phi: \mathbb{D} \stackrel{\text { onto }}{\longrightarrow} \mathbb{D}$ is a homeomorphism of the same distortion function, $K_{\Phi}(z)=K_{G}(z)$. Moreover, $\mathcal{H}: \mathbb{D} \rightarrow \mathbb{C}$ is holomorphic.

Here are additional properties of $\Phi$ :

(i) $\Phi \in \mathscr{W}^{1,1}(\mathbb{D})$

(ii) $\Phi^{-1} \in \mathscr{W}^{1,2}(\mathbb{D})$

For (i) we argue as follows, $|D \Phi(z)|^{2} \stackrel{\text { def }}{=} K_{\Phi}(z) J_{\Phi}(z)=K_{G}(z) J_{\Phi}(z)$. Hence, by Hölder's inequality,

$$
\left(\int_{\mathbb{D}}|D \Phi(z)| \mathrm{d} z\right)^{2} \leqslant\left(\int_{\mathbb{D}} K_{G}(z) \mathrm{d} z\right) \cdot \int_{\mathbb{D}} J_{\Phi}(z) \mathrm{d} z=\pi \int_{\mathbb{D}} K_{G}(z) \mathrm{d} z<\infty
$$

For (ii) we make use of change of variables $w=\Phi(z)$. Accordingly, $D \Phi^{-1}(w)=[D \Phi(z)]^{-1}=D^{\sharp} \Phi(z) / J_{\Phi}(z)$, where $D^{\sharp} g$ stands for the cofactor matrix of $D \Phi$. Hence

$$
\begin{aligned}
& \int_{\mathbb{D}}\left|D \Phi^{-1}(w)\right|^{2} \mathrm{~d} w=\int_{\mathbb{D}}\left|\frac{D^{\sharp} \Phi(z)}{J_{\Phi}(z)}\right|^{2} J_{\Phi}(z) \mathrm{d} z=\int_{\mathbb{D}} K_{\Phi}(z) \mathrm{d} z \\
&=\int_{\mathbb{D}} K_{G}(z) \mathrm{d} z<\infty \\
& 12
\end{aligned}
$$


Stoilow's factorization of $G$ in Proposition 8 yields a Stoilow factorization of $F$; namely,

$$
F(z)=\mathcal{H}(w), \quad \text { where } w=w(z) \stackrel{\text { def }}{=} \Phi\left(|z|^{\frac{1-\varepsilon}{\varepsilon}} z\right)
$$

Clearly, $w: \mathbb{D} \stackrel{\text { onto }}{\longrightarrow} \mathbb{D}$ is a homeomorphism. Proceeding further in this direction, we note that every holomorphic function $\mathcal{H}$ in $\mathbb{D}$ which vanishes only at the origin can be written as $\mathcal{H}(w)=[\mathcal{P}(w)]^{m}$, for some holomorphic function $\mathcal{P}: \mathbb{D} \rightarrow \mathbb{C}$ with $\mathcal{P}^{\prime}(0) \neq 0$. Here $m$ is the order of zero of $\mathcal{H}$. Thus $\mathcal{P}$ is a conformal homeomorphism near the origin. We summarize with a local representation of $F$ in a spirit similar to that of Proposition 7.

Proposition 9. . Recall, we conveniently confined ourselves to $\Omega=$ $\mathbb{D}=\{z \in \mathbb{C}:|z|<1\}$. For all $z \in \Omega$, we have

$$
F(z)=[\Theta(z)]^{m}
$$

Here $\Theta \stackrel{\text { def }}{=} \mathcal{P} \circ w: \Omega \rightarrow \mathbb{C}$ is an orientation preserving homeomorphisms defined near the origin, $\Theta(0)=0$. Actually, $\Theta$ is a $\mathscr{C}^{\infty}$. diffeomorphism in $\Omega_{\circ}=\Omega \backslash\{0\}$.

Corollary 10. Near a critical point at $0 \in \Omega$, where $u(0)=0$, the level set $\mathcal{L}_{u}=\{z: u(z)=0\}$ consists of $2 m$ Jordan arcs emanating from 0 . Precisely,

$$
\begin{gathered}
\mathcal{L}_{u}=\ell_{1} \cup \ell_{2} \cup \cdots \cup \ell_{2 m} \\
\text { where } \ell_{k}=\Theta^{-1}\left(L_{k}\right), L_{k} \stackrel{\text { def }}{=}\left\{t e^{\frac{2 k-1}{2 m} \pi i}: 0 \leqslant t<\rho\right\}, k=1, \ldots, 2 m .
\end{gathered}
$$

Later we shall show that $m=n+1$, where $n \geqslant 1$ is defined via Stoilow's factorization of the complex gradient of $u$, see (2.6). The proof will follow very closely a computation by G. Aronsson and P. Lindqvist [8, Theorem 6, p. 166-167], see also the subsequent papers [1, 2 ] concerning the critical points of $p$-harmonic functions. The idea in [8] is worth the effort of presenting it in details. Sections 4 and 5 are devoted to necessary preliminaries.

\section{INCREMENT OF ARGUMENTS}

Throughout this section $\Gamma \subset \mathbb{C}$ will be an oriented Jordan arc with endpoints, or a simple closed curve oriented counterclockwise

$$
\Gamma=\{z(t) ; a \leqslant t \leqslant b\} \subset \mathbb{R}^{2}, \quad \text { for a simple closed curve } z(a)=z(b)
$$

Thus the point $z(t)$ moves continuously along $\Gamma$ in positive direction as parameter $t$ increases. Consider a continuous (nonvanishing) vector 


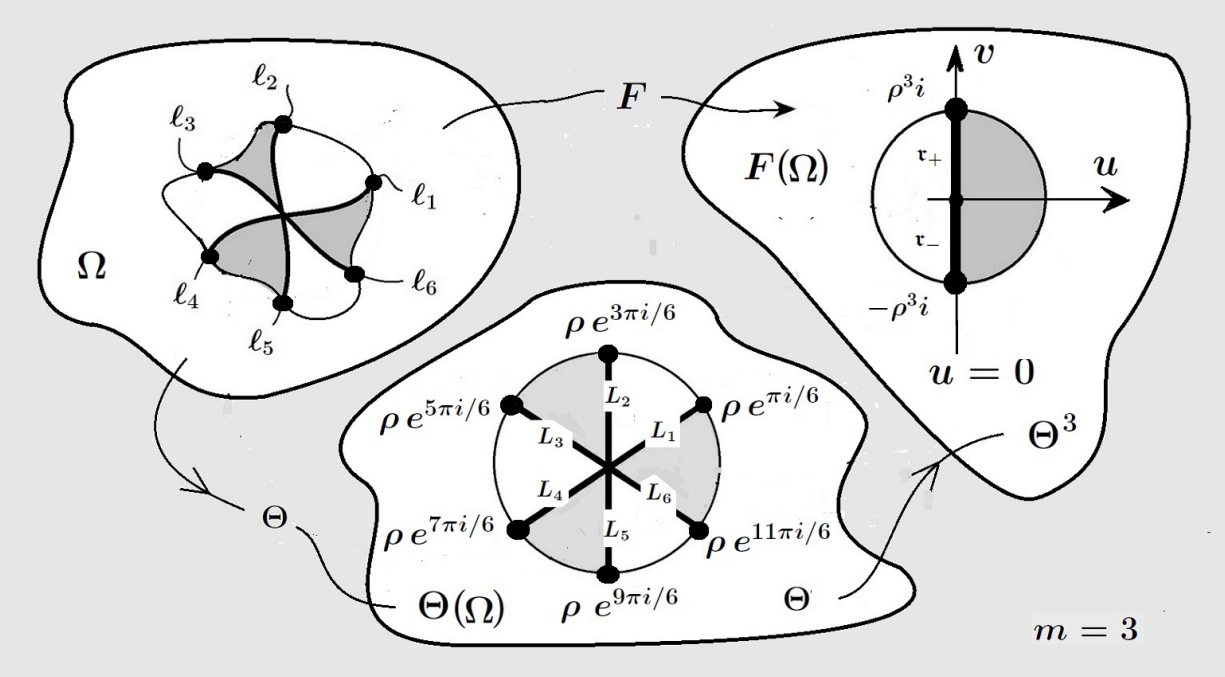

Figure 2. The level curves $\ell_{1}, \ldots, \ell_{6}$, emanating from a critical point, are rectified by a homeomorphism $\Theta=$ $\Theta(z)$ to become straight rays $L_{1}, \ldots, L_{6}$ in a disk. Then the conformal cubic map $\Theta \rightarrow \Theta^{3}$ takes them into two vertical rays, $\mathfrak{r}_{+}$and $\mathfrak{r}_{-}$.

field $\mathbf{V}: \Gamma \rightarrow \mathbb{C}_{\circ}$. To every parameter $t \in[a, b]$ there corresponds a countable family of arguments of the complex number $\mathbf{V}(z(t)) \in \mathbb{C}_{0}$. We may (and do) choose a continuous branch of arguments of $\mathbf{V}(z(t))$ as $t$ varies from $a$ to $b$. Denote it by Arg : $[a, b] \stackrel{\text { into }}{\longrightarrow} \mathbb{R}$ and define the increment of arguments of $\mathbf{V}$ along $\Gamma$ by setting

$$
\Delta_{\Gamma}(\arg \mathbf{V})=\operatorname{Arg}(b)-\operatorname{Arg}(a)
$$

This definition is legitimate because it does not depend on the choice of the branch Arg. One basic property of this concept should be pointed out. Suppose we divide $\Gamma$ into closed sub-arcs, $\Gamma=\Gamma_{1} \cup \Gamma_{2} \cup \ldots \cup \Gamma_{\ell}$, in which every sub-arc has its own parametrization and its own continuous branch of arguments of $\mathbf{V}$. Then

$$
\Delta_{\Gamma}(\arg \mathbf{V})=\Delta_{\Gamma_{1}}(\arg \mathbf{V})+\Delta_{\Gamma_{2}}(\arg \mathbf{V})+\ldots+\Delta_{\Gamma_{\ell}}(\arg \mathbf{V})
$$

Here it is important that $\mathbf{V}$ is non-vanishing and continuous along $\Gamma$.

4.1. Tangent Vector Field. Let $\Gamma$ be a $\mathscr{C}^{1}$-smooth simple closed curve. Thus $\Gamma$ surrounds a simply connected Jordan domain $\Omega$. We assume that $\Gamma$ is positively oriented so that traveling along $\Gamma$ in positive direction (counterclockwise) the domain remains on the left hand 
side. Thus we have well defined continuous unit tangent vector field $\mathbf{T}: \Gamma \rightarrow \mathbb{S}^{1}, \quad \mathbf{T}(z(t))=\frac{\dot{z}(t)}{|\dot{z}(t)|}$.

Proposition 11. The increment of the tangent argument $\Delta_{\Gamma}(\arg \mathbf{T})$ equals $2 \pi$.

Proof. Let $\gamma:[a, b] \rightarrow \mathbb{C}$ be an arclength parametrization of a $\mathscr{C}^{2}$ smooth oriented curve $\Gamma$. Thus the unit tangent vector field $\gamma^{\prime}=$ $\gamma^{\prime}(s) \neq 0, a \leqslant s \leqslant b$, represents the orientation of $\Gamma$. We express it by the formula $\gamma^{\prime}(s)=e^{i \theta(s)}$. The derivative of the angle $\theta=\theta(s)$ defined along the interval $[a, b]$, gives well defined signed curvature of $\Gamma, \kappa(s) \stackrel{\text { def }}{=} \theta^{\prime}(s)$. The tangent increment along $\Gamma$ is none other than $\int_{a}^{b} \kappa(s) \mathrm{d} s$. The integral $\int_{a}^{b} \kappa(s) \mathrm{d} s$ is known as the total signed curvature.

There is an extensive literature dealing with the concept of total signed curvature and its higher dimensional counterparts. The following statement can be viewed as a special case of the Gauss-Bonnet theorem [20, $44]$.

Theorem 12. The total signed curvature of a smooth closed curve, $\gamma(a)=\gamma(b)$, in the plane is a topological invariant. More precisely, $\int_{a}^{b} \kappa(s) \mathrm{d} s$ does not change within a smooth homotopy deformation of $\Gamma$.

This result, sometimes known as the Hopf winding number theorem says, in particular, that every positively oriented simple closed curve in the plane has total signed curvature equal to $2 \pi$.

It is appropriate at this point to mention a paper by J. Malý [39] in which the tangent increment is studied for closed curves with selfintersections.

Remark 13. Given $\mathscr{C}^{1}$-arc, or a simple closed curve, there are two continuous unit tangent fields, $\mathbf{T}: \Gamma \rightarrow \mathbb{S}^{1}$ and $-\mathbf{T}: \Gamma \rightarrow \mathbb{S}^{1}$. It may seem counterintuitive, but in fact both have the same increment of argument. Indeed, regardless of the choices of continuous branches of argument for $\mathbf{T}(z(t))$ say $\mathbf{T}(z(t))=e^{i \theta(t)}$, we have $-\mathbf{T}(z(t))=$ $e^{i(\theta(t)+\pi)}$. Thus their arguments differ by a constant independent of the point $z(t) \in \Gamma$.

Remark 14. On the other hand, given a continuous (nonvanishing) vector field $\mathbf{V}=a+i b: \Gamma \rightarrow \mathbb{C}_{\circ}$ the increment of its complex conjugate field $\overline{\mathbf{V}}=a-i b: \Gamma \rightarrow \mathbb{C}_{\circ}$ changes sign

$$
\Delta_{\Gamma} \arg \overline{\mathbf{V}}=-\Delta_{\Gamma} \arg \mathbf{V}
$$


This observation is immediate from the formula

$$
\mathbf{V}(z)=\rho(z) e^{i \theta(z)} \Longrightarrow \overline{\mathbf{V}}(z)=\rho(z) e^{-i \theta(z)} \quad \text { for every } z \in \Gamma .
$$

4.2. Curved Polygons. Consider a simply connected Jordan domain $\Omega \subset \mathbb{C}$ whose boundary $\Gamma=\partial \Omega$ (a simple closed curve oriented counterclockwise) contains $N$ distinct points $C_{1}, C_{2}, \ldots, C_{N}, N \geqslant 2$, called corners of $\Omega$. One meets these points in the above order when traveling in the positive direction along $\Gamma$. Consider the compact Jordan arcs (with endpoints) connecting two consecutive corners

$$
\Gamma_{1} \stackrel{\text { def }}{=}\left[C_{1} \rightsquigarrow C_{2}\right], \ldots, \Gamma_{\ell} \stackrel{\text { def }}{=}\left[C_{\ell} \rightsquigarrow C_{\ell+1}\right], \ldots, \Gamma_{N} \stackrel{\text { def }}{=}\left[C_{N} \rightsquigarrow C_{1}\right]
$$

We assume that each $\Gamma_{\ell}$ is $\mathscr{C}^{1}$-smooth up to its endpoints. To make this assumption precise, we parametrize $\Gamma_{\ell}$ as

$$
\Gamma_{\ell}=\left\{z_{\ell}(t) ; 0 \leqslant t \leqslant 1\right\}, \text { where } z_{\ell}(1)=z_{\ell+1}(0)=C_{\ell+1}
$$

Hereafter, we adhere to the convention that $z_{N+1}(t)=z_{1}(t)$. In particular $C_{N+1}=C_{1}$ and $\Gamma_{N+1}=\Gamma_{1}$. Thus, we actually assume that the complex-valued functions $z_{\ell} \in \mathscr{C}^{1}[0,1]$ have nonvanishing derivative in the closed interval $[0,1]$, where

$$
\dot{z}_{\ell}(0) \stackrel{\text { def }}{=} \lim _{t \searrow 0} \dot{z}_{\ell}(t) \quad \text { and } \quad \dot{z}_{\ell}(1) \stackrel{\text { def }}{=} \lim _{t \nearrow 1} \dot{z}_{\ell}(t)
$$

A continuous unit tangent vector field on $\Gamma_{\ell}$ is given by

$$
T^{\ell}=T^{\ell}(t)=\frac{\dot{z}_{\ell}(t)}{\left|\dot{z}_{\ell}(t)\right|} \in \mathbb{S}^{1}
$$

At every corner $C_{\ell}$ we have two unit vectors,

$$
\begin{gathered}
T_{-}^{\ell}=\frac{\dot{z}_{\ell-1}(1)}{\left|\dot{z}_{\ell-1}(1)\right|} \quad\left(\begin{array}{c}
\text { tangent to } \Gamma_{\ell-1} \text { at } \\
\text { its destination point }
\end{array}\right), \\
T_{+}^{\ell}=\frac{\dot{z}_{\ell}(0)}{\left|\dot{z}_{\ell}(0)\right|} \quad\left(\begin{array}{c}
\text { tangent to } \Gamma_{\ell} \text { at } \\
\text { its starting point }
\end{array}\right)
\end{gathered}
$$

The case $T_{-}^{\ell}=T_{+}^{\ell}$ simply means that $\Gamma_{\ell-1} \cup \Gamma_{\ell}$ is a $C^{1}$-smooth arc. In this case the point $C_{\ell} \in \Gamma$ can be viewed as a corner of zero angle, see below the definition of the angle. Nevertheless, whenever speaking of $N$ as the number of corners we mean sharp corners; that is, $T_{-}^{\ell} \neq T_{+}^{\ell}$ for every $\ell=1,2, \ldots, N$. In this case $\left.\left[\Omega ; C_{1}, \ldots, C_{N}\right)\right]$ is referred to as a curved $N$-polygon with (sharp) corners $C_{1}, \ldots, C_{N}$. Its sides $\Gamma_{1}, \ldots \Gamma_{N}$ are $\mathscr{C}^{1}$-smooth arcs. Such an oriented (counterclockwise) curved polygon has uniquely defined angle $\alpha_{\ell} \in[-\pi, \pi]$ at the corner 
$C_{\ell}$. For this, we first consider a cusp at $C_{\ell}$ and set $\alpha_{\ell}=-\pi$ for the inward cusp and $\alpha_{\ell}=\pi$ for the outward cusp. Otherwise, we define

$$
\alpha_{\ell}=\operatorname{Arg} \frac{\dot{z}_{\ell}(0)}{\dot{z}_{\ell-1}(1)} \in(-\pi,+\pi) \text {. Thus } T_{+}^{\ell}=e^{i \alpha_{\ell}} T_{-}^{\ell}
$$

The vector $T_{+}^{\ell}$ is obtained by rotating $T_{-}^{\ell}$; counterclockwise if $\alpha_{\ell}>0$ and clockwise if $\alpha_{\ell}<0$.

Let us take a quick look at an $N$-polygon whose sides are straight line segments. Thus, we have no cusps. It follows from the definition of $\alpha_{\ell}$ that $\beta_{\ell} \stackrel{\text { def }}{=} \pi-\alpha_{\ell} \in(0,2 \pi)$ is the angle inside the polygon at which the sides $\Gamma_{\ell-1}$ and $\Gamma_{\ell}$ meet. Call it the interior angle of the polygon at $C_{\ell}$. The elementary geometric considerations show that

$$
\beta_{1}+\beta_{2}+\ldots+\beta_{N}=(N-2) \pi, \quad \alpha_{1}+\alpha_{2}+\ldots+\alpha_{N}=2 \pi
$$

Since the sides are straight line segments, their tangent vector fields $T^{\ell}$ are constant, so $\Delta_{\Gamma_{\ell}} \arg T^{\ell}=0$. Thus, formally,

$$
\left(\alpha_{1}+\alpha_{2}+\ldots+\alpha_{N}\right)+\left(\Delta_{\Gamma_{1}} \arg T^{1}+\ldots+\Delta_{\Gamma_{N}} \arg T^{N}\right)=2 \pi
$$

A generalization of this geometric observation reads as follows.

Proposition 15. Formula (4.5) remains valid for all curved $N$-polygons. In this general formula the increments $\Delta_{\Gamma_{\ell}} \arg T^{\ell}$ may not vanish.

Proof. The idea is to round out the corners of $\Gamma$ and apply Proposition 11 for smooth curve. For every sufficiently small $\varepsilon>0$ we construct a $\mathscr{C}^{1}$-smooth Jordan curve $\Gamma^{\varepsilon}$ in close proximity to $\Gamma$. To this effect, consider two adjacent sides $\Gamma_{\ell-1}$ and $\Gamma_{\ell}$ which meet at the corner $C_{\ell}$ . Near this corner one can draw arbitrarily small open disk $\mathbb{D}_{\ell} \subset \mathbb{C} \backslash \Gamma$ of radius $\leqslant \varepsilon$, which is tangent to both $\Gamma_{\ell-1}$ and $\Gamma_{\ell}$. There is no need to have the radius of $\mathbb{D}_{\ell}$ exactly equal to $\varepsilon$. Such a disk lies either in $\Omega$ or outside $\Omega$. The first case occurs when $\alpha_{\ell}>0$ and the second when $\alpha_{\ell}<0$. The construction of $\mathbb{D}_{\ell}$ as a nice exercise in elementary geometry. Denote by $\gamma_{\ell}^{\varepsilon} \subset \partial \mathbb{D}_{\ell}$ the shortest oriented circular arc whose starting point lies in $\Gamma_{\ell-1}$ and destination point lies in $\Gamma_{\ell}$. Thus at the points of tangency the orientation of the arc $\gamma_{\ell}^{\varepsilon}$ agrees with that of $\Gamma$. Precisely, the orientation of $\gamma_{\ell}^{\varepsilon}$ is counterclockwise if $\alpha_{\ell}>0$ and clockwise if $\alpha_{\ell}<0$.

Replace the sharp fragment of $\Gamma$ near $C_{\ell}$ by $\gamma_{\ell}^{\varepsilon}$. In this way we arrive at a $\mathscr{C}^{1}$-smooth counterclockwise oriented Jordan closed curve, denoted by $\Gamma^{\varepsilon}$. 


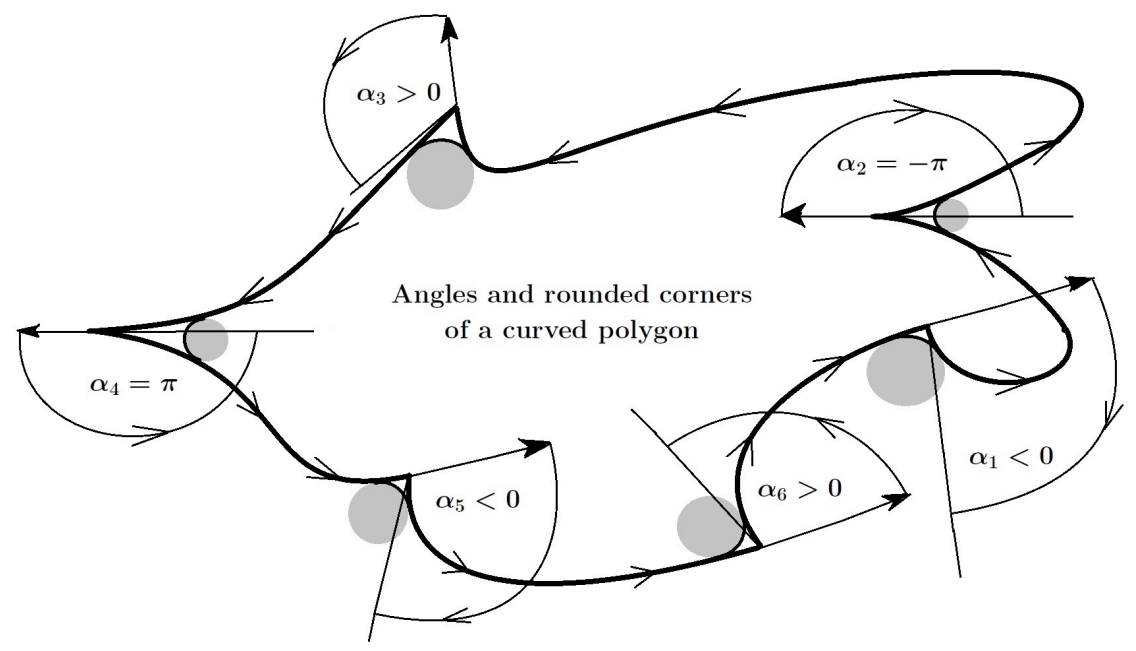

FiguRE 3. The circular arcs of small disks tangent to $\Gamma$. We replace the sharp corners of $\Gamma$ by the circular arcs to form a $\mathscr{C}^{1}$-smooth curve $\Gamma^{\epsilon}$.

Let $\mathbf{T}^{\varepsilon}$ denote the unit tangent vector field along $\Gamma^{\varepsilon}$. Thus $\mathbf{T}^{\varepsilon}=T^{\ell}$ on $\Gamma_{\ell}^{\varepsilon} \stackrel{\text { def }}{=} \Gamma_{\ell} \cap \Gamma^{\varepsilon}$. For every, sufficiently small, $\varepsilon$ we have

$$
\sum_{\ell}^{N} \Delta_{\gamma_{\ell}^{\varepsilon}}\left(\arg \mathbf{T}^{\varepsilon}\right)+\sum_{\ell}^{N} \Delta_{\Gamma_{\ell}^{\varepsilon}}\left(\arg \mathbf{T}^{\varepsilon}\right)=\Delta_{\Gamma^{\varepsilon}}\left(\arg \mathbf{T}^{\varepsilon}\right)=2 \pi
$$

by Proposition 11. In the above sum each term has well defined limit as $\varepsilon \rightarrow 0$. First, since $T^{\ell}$ is continuous on $\Gamma_{\ell}$ up to its endpoints,

$$
\lim _{\varepsilon \rightarrow 0} \Delta_{\Gamma_{\ell}^{\varepsilon}}\left(\arg \mathbf{T}^{\varepsilon}\right)=\Delta_{\Gamma_{\ell}}\left(\arg T^{\ell}\right)
$$

For circular arcs, on the other hand, we have

$$
\lim _{\varepsilon \rightarrow 0} \Delta_{\gamma_{\ell}^{\varepsilon}} \arg \mathbf{T}^{\varepsilon}=\alpha_{\ell}
$$

Now Formula (4.5) follows from (4.6) after passage to the limit as $\varepsilon \rightarrow 0$.

\section{The CuRved $4 m$-POlygon AROUnd the CRITICAL POINT}

We return to $F=u+i v=[\Theta(z)]^{m}$ in formula (3.5) and a homeomorphism $\Theta: \Omega \stackrel{\text { onto }}{\longrightarrow} \Omega^{\prime} \stackrel{\text { def }}{=} \Theta(\Omega)$, where $\Theta(0)=0$. Recall that $\Theta$ is a $\mathscr{C}^{\infty}$-diffeomorphism of $\Omega_{\circ}$ onto $\Omega_{\circ}^{\prime}$ and that the aim is to show that $m=n+1$. 
With this purpose in mind we may assume, by rescaling if necessary, that the target $\Omega^{\prime}$ contains the closed disk

$$
Q \stackrel{\text { def }}{=}\left\{(u, v):|u| \leqslant \frac{\sqrt{2}}{2} \text { and }|v| \leqslant \frac{\sqrt{2}}{2}\right\} \Subset \Omega^{\prime}
$$

Consider points $\xi_{\ell} \stackrel{\text { def }}{=} \exp \frac{(2 \ell-1) \pi i}{4 m} \in \mathbb{S}^{1}, \ell=1,2, \ldots, 4 m$, so their $m$-th powers are corners of $Q$. Namely, $\xi_{\ell}^{m}=( \pm 1 \pm i) \frac{\sqrt{2}}{2}$. We then connect the consequent corners $\xi_{\ell-1}$ and $\xi_{\ell}$ with a Jordan arc whose image under the power map $\xi \rightarrow \xi^{n}$ is a straight line side of $Q$. Connecting the corners $\xi_{\ell-1}^{m}$ with $\xi_{\ell}^{m}$. in this way we obtain "regular" curved $4 m$ polygon, denoted by $\mathbb{P}_{4 m}$. Note that all angles at the corners of $\mathbb{P}_{4 m}$ are equal to $\frac{\pi}{2}$, because the power map is orientation preserving and conformal, see Figure 4.

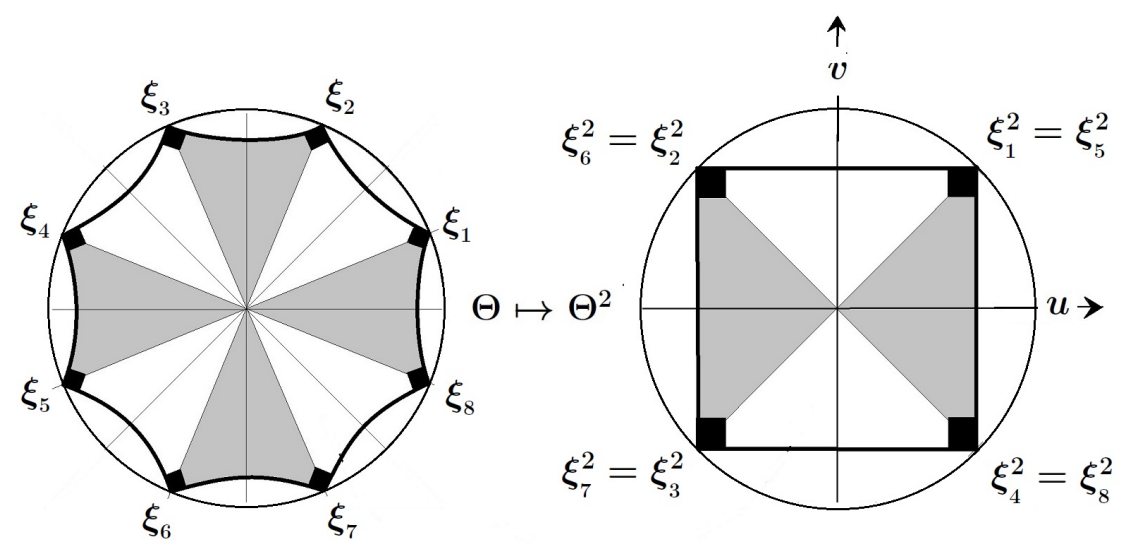

Figure 4 . The conformal map $\xi \rightarrow \xi^{2}$ takes a curved 8-polygon with right angles at $\xi_{1}, \ldots, \xi_{8}$ onto a square.

Next denote by $\mathbb{G}_{4 m}=\Theta^{-1}\left(\mathbb{P}_{4 m}\right) \Subset \Omega$ the preimage of $\mathbb{P}_{4 m}$ under the (orientation preserving) homeomorphism $\Theta: \Omega \stackrel{\text { onto }}{\longrightarrow} \Theta(\Omega)$ and $\Gamma=\partial \mathbb{G}_{4 m}$. Thus $\Theta$ takes a neighborhood of $\Gamma$ onto a neighborhood of $\partial \mathbb{P}_{4 m}$. In this way $\Gamma$ itself becomes an oriented curved $4 m$-polygon. Following our notation in (4.3), we let $C_{1}, C_{2}, \ldots, C_{4 m}$ and $\Gamma_{1}, \Gamma_{2}, \ldots, \Gamma_{4 m}$ denote the corners and sides of $\Gamma$, respectively.

Lemma 16. The oriented angles $\alpha_{1}, \alpha_{2}, \ldots, \alpha_{4 m}$ at the corners of $\Gamma$, defined by (4.4) are also equal to $\frac{\pi}{2}$. 


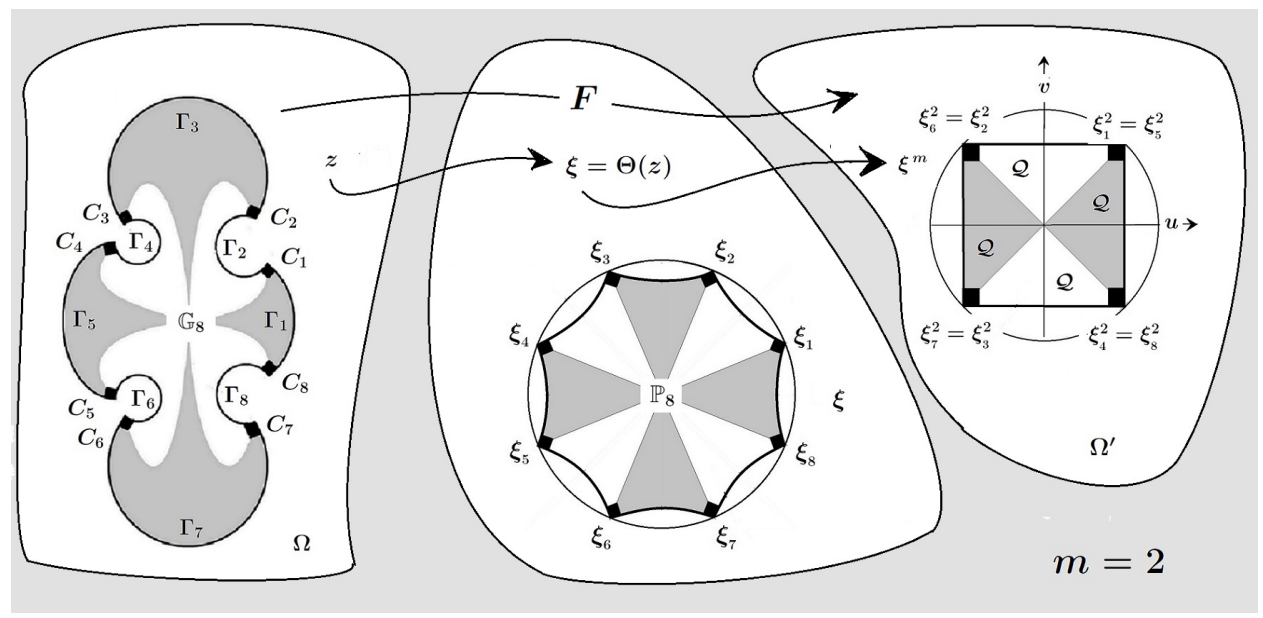

Figure 5. Tranformation of a curved 8-polygon $\mathbb{G}_{8}$ onto a regular 8-polygon $\mathbb{P}_{8}$ and then onto a square.

Proof. But the reasoning is not via transformation. We argue as follows. The adjacent sides of $\mathbb{G}_{4 m}$ meet at the right angle we argue as follows. The map $F=u+i v=[\Theta(z)]^{m}$ takes $\mathbb{G}_{4 m}$ onto the square $Q$ (covers it $m$-times). Each side $\Gamma_{\ell}$ of $\Gamma=\partial \mathbb{G}_{4 m}$ is mapped onto one of the sides of the square. Hence, one (and only one) of the coordinate functions $u$ or $v$ is constant along $\Gamma_{\ell}$ (equal to $\pm \frac{\sqrt{2}}{2}$ ). The other coordinate varies between $-\frac{\sqrt{2}}{2}$ and $\frac{\sqrt{2}}{2}$. These properties of $u$ and $v$ alternate when passing from $\Gamma_{\ell}$ to $\Gamma_{\ell+1}$. As always, the gradient $\nabla u$ is orthogonal to the level curves of $u$. Similarly, $\nabla v$ is orthogonal to the level curves of $v$. On the other hand $\nabla u$ and $\nabla v$ are orthogonal everywhere in $\Omega$, because they satisfy the same $p$-harmonic equation because $u$ and $v$ satisfy the $(p, q)$ system (3.1). Hence it is readily seen that the sides $\Gamma_{\ell}$ and $\Gamma_{\ell+1}$ meet at the corner of $\Gamma$ perpendicularly. In terms of the oriented angles $\alpha_{1}, \alpha_{2}, \ldots, \alpha_{4 m}$, defined by (4.4), this means $\alpha_{\ell}= \pm \frac{\pi}{2}$, for all $\ell=1,2, \ldots, 4 m$. The possibility $\alpha_{\ell}=-\frac{\pi}{2}$ is ruled out as follows. The orientation preserving $\mathscr{C}^{1}$ - diffeomorphism $\Theta$ takes a neighborhood of $\partial \mathbb{G}_{4 m}$ onto a neighborhood of $\partial \mathbb{P}_{4 m}$. The interior angles at the corners of $\mathbb{P}_{4 m}$ are equal to $+\frac{\pi}{2}$. Now the following geometric observation tells us that the angles $\alpha_{1}, \alpha_{2}, \ldots, \alpha_{4 m}$ are equal to $\frac{\pi}{2}$.

Lemma 17. Suppose that a curved $N$-polygon $\Gamma$ is deformed onto $N$ polygon $\Gamma^{\prime}$ via a $\mathscr{C}^{1}$-diffeomorphism defined in a neighborhood of $\Gamma$. Then every angle $\alpha_{\ell}$ of $\Gamma$ and the corresponding angle $\alpha_{\ell}^{\prime}$ of $\Gamma^{\prime}$ have the same sign, $\ell=1,2, \ldots, N$. 
Proof. This is actually a local fact so we need only consider a $\mathscr{C}^{1}$ deformation near a given corner $C_{\ell}$ of $\Gamma$. Note that $\alpha_{\ell}>0$ if and only if the interior angle $\beta_{\ell}=\pi-\alpha_{\ell}$ belongs to $(0, \pi)$. Suppose, to the contrary, that $\alpha_{\ell}$ and $\alpha_{\ell}^{\prime}$ have different sign. Then one of the angles $\beta_{\ell}=\pi-\alpha_{\ell}$ or $\beta_{\ell}^{\prime}=\pi-\alpha_{\ell}^{\prime}$ would lie in $(0, \pi)$ while the other would lie in $(\pi, 2 \pi)$. But this is impossible. Just look at the linear tangent map of the diffeomorphism in question. We are reduced to a nonsingular linear transformation of a straight line angular sector to another straight line angular segment. Such a linear map cannot convert an angular segment of opening $<\pi$ onto a segment of opening $>\pi$, see Figure 6

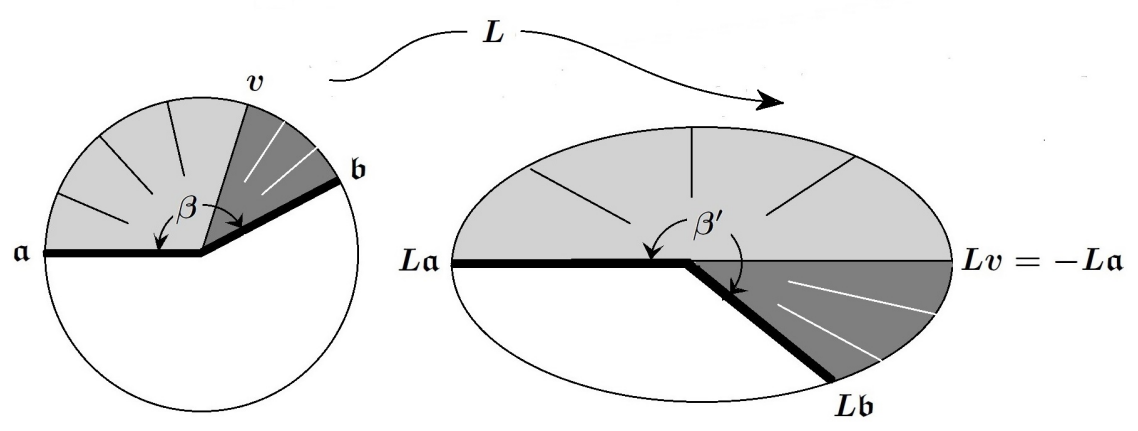

Figure 6. It is impossible to make $0<\beta<\pi<\beta^{\prime}<2 \pi$ by a linear transformation $L: \mathbb{R}^{2} \rightarrow \mathbb{R}^{2}$. Otherwise $L$ would take two independent vectors $\mathfrak{a}$ and $v$ into two linearly dependent vectors $L \mathfrak{a}$ and $-L \mathfrak{a}$.

\section{The RELATIONShiP $m=n+1$}

Recall $m$ and $n$ from Proposition 9 and Proposition 7, respectively.

Proposition 18. We have

$$
m=n+1 \geqslant 2
$$

In particular, the number $2 m=2 n+2$ of the level arcs emanating from a critical point equals 4 at least. For completeness, the number of level arcs amanating from a regular point equals 2

Proof. We invoke to the curved polygon $\mathbb{G}_{4 m}$ and its boundary $\Gamma=$ $\partial \mathbb{G}_{4 m}=\Gamma_{1} \cup \Gamma_{2} \cup \cdots \cup \Gamma_{4 m}$ in Section 5. The gradient $\nabla u$ is a continuous vector field along $\Gamma$. It is either tangent or normal on 
every side $\Gamma_{\ell}, \ell=1, \ldots, 4 m$. This property reverses in passing from $\Gamma_{\ell}$ to $\Gamma_{\ell+1}$. We see that on each side $\Gamma_{\ell}$ (no matter if $\nabla u$ is tangent or normal to $\Gamma_{\ell}$ ) every continuous branch of arguments of $\nabla u$ differs by a constant from a continuous branch of arguments of the tangent field. Thus

$$
\Delta_{\Gamma_{\ell}} \arg \nabla u=\Delta_{\Gamma_{\ell}} \arg T^{\ell}, \quad \text { for every } \ell=1,2, \ldots, 4 m
$$

We now appeal to formula (4.5) with $\alpha_{\ell}=\frac{\pi}{2}$. This yields

$$
2 \pi m+\Delta_{\Gamma_{1}} \arg \nabla u+\Delta_{\Gamma_{2}} \arg \nabla u+\ldots+\Delta_{\Gamma_{4 m}} \arg \nabla u=2 \pi
$$

Since $\nabla u$ is continuos (and nonvanishing) along $\Gamma$, it holds that

$$
\Delta_{\Gamma_{1}} \arg \nabla u+\Delta_{\Gamma_{2}} \arg \nabla u+\ldots+\Delta_{\Gamma_{4 m}} \arg \nabla u=\Delta_{\Gamma} \arg \nabla u
$$

Hence $\Delta_{\Gamma} \arg \nabla u=2(1-m) \pi$. Concerning the complex conjugate field $f=\frac{1}{2}\left(u_{x}-i u_{y}\right)$, by Remark 14 , we obtain

$$
\Delta_{\Gamma} \arg f=-\Delta_{\Gamma} \arg \left(u_{x}+i u_{y}\right)=-\Delta_{\Gamma} \arg \nabla u=2(m-1) \pi
$$

This combined with formula (2.6) gives $\Delta_{\Gamma} \arg f=2 \pi n$. Hence $m=n+1$, as desired.

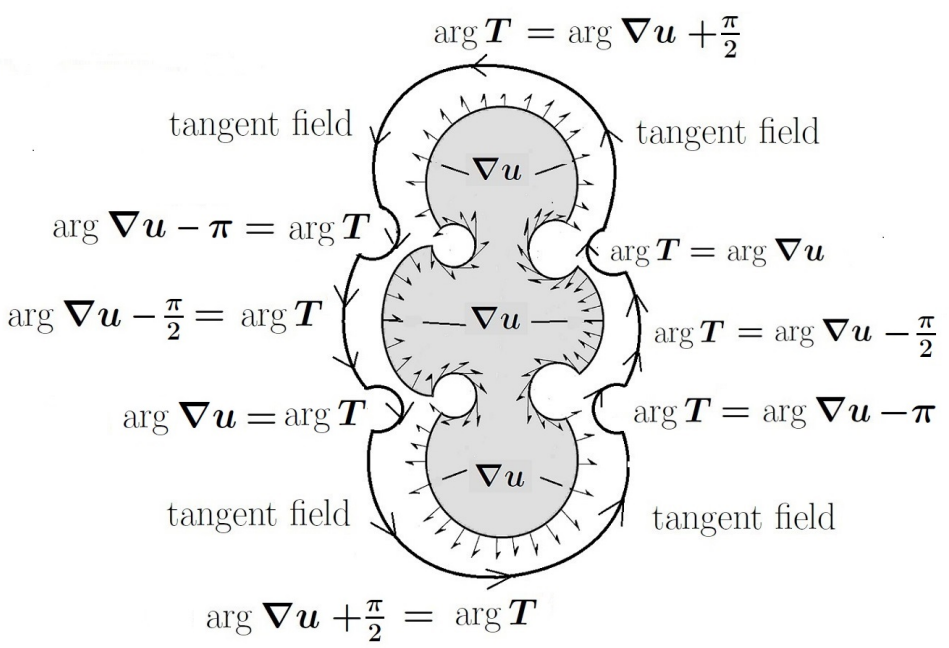

FiguRE 7. Along $\Gamma$ the continuous gradient field $\nabla u$ is either tangent or normal on every side of $\Gamma$. 


\section{The $p$-HARMonic Dendrite}

Recall a nonconstant $p$-harmonic function $u: \Omega \rightarrow \mathbb{R}$ defined in a simply-connected domain $\Omega \subset \mathbb{R}^{2}$, its level set $\mathfrak{L} \stackrel{\text { def }}{=}\{z \in \Omega ; u(z)=$ $0\}$, and the discrete set $\{z ; \nabla u(z)=0\}$, of critical points. Thus $\nabla u$ does not vanish in the punctured domain $\Omega_{\circ} \stackrel{\text { def }}{=}\{z ; \nabla u(z) \neq 0\}$. It follows from the local structure of the level set, described in detail by Corollary 10, that

(a) $\mathfrak{L}$ is locally path connected.

Proof. For thorough arguments, we introduce the so-called branching neighborhoods $\mathcal{B}_{z}(\varepsilon)$ of a point $z \in \mathfrak{L}$. Let $z=0 \in \mathfrak{L}$. Corollary 10 combined with Proposition 18 tells us that from every point $z \in \mathfrak{L}$ there emanate $2 k$ Jordan arcs $\ell_{1}, \ell_{2}, \ldots, \ell_{2 k} \subset \mathfrak{L}, 1 \leqslant k<\infty$. Precisely, $k=1$ if $z$ is a regular point and $k=2 m \geqslant 4$ when $z$ is a critical point. The branching neighborhoods are defined by the rule $\mathcal{B}_{z}(\varepsilon) \stackrel{\text { def }}{=} \Theta^{-1}\left(\mathbb{D}_{\varepsilon}\right)$, where $\mathbb{D}_{\varepsilon}=\{z:|z|<\varepsilon\}$ and $\varepsilon>0$ is sufficiently small. Clearly, the set $\mathfrak{L} \cap \mathcal{B}_{z}(\varepsilon)$ are path connected. In fact, every point in $\mathfrak{L} \cap \mathcal{B}_{z}(\varepsilon)$ can be connected with $z$ by a path in $\mathfrak{L} \cap \mathcal{B}_{z}(\varepsilon)$.

(b) $\mathfrak{L}$ contains no simple closed Jordan arc.

Proof. Suppose, to the contrary, that there is a simple closed Jordan arc $\gamma \subset \mathfrak{L}$ on which $u$ vanishes. By Jordan Closed Curved Theorem, since $\Omega$ is simply connected, the bounded complementary domain of $\gamma$ lies in $\Omega$. Then by the maximum/minimum principle, $u$ vanishes in this domain. By unique continuation principle $u$ vanishes in $\Omega$, contrary to the assumption that $u \neq \equiv 0$.

The above two properties $(a)$ and $(b)$ motivate our calling $\mathfrak{L}$ a $p$ harmonic dendrite. Note that $\mathfrak{L}$ is neither compact nor (in general) connected.

(c) $\mathfrak{L}$ can be covered by a locally finite family of branching neighborhoods, say

$$
\mathscr{B}=\left\{\mathfrak{B}_{z_{1}}, \mathfrak{B}_{z_{2}}, \ldots\right\}
$$

Proof. Begin with a cover $\mathfrak{L} \subset \bigcup_{z \in \mathfrak{L}} \mathfrak{B}_{z}$ and select a locally finite subcover $\mathscr{B}=\left\{\mathfrak{B}_{z_{1}}, \mathfrak{B}_{z_{2}}, \ldots\right\}$.

We may, and do, include to $\mathscr{B}$ branching neighborhoods of critical points, so that $\left\{z_{1}, z_{2}, \ldots\right\}$ contains all critical points. Once such $\mathscr{B}$ is selected and fixed, we refer to $z_{1}, z_{2}, \ldots$, as the branching points of the dendrite. It has to be pointed out that some branching points need 
not be critical points; exactly those from which there emanate two half open $\operatorname{arcs} \ell_{1}, \ldots, \ell_{2 k}, k=1$.

(d) Fix a point $z_{\circ} \in\left\{z_{1}, z_{2}, \ldots,\right\}$ and let $\ell_{1}, \ldots, \ell_{2 k}$ be the half open arcs which emanate from $z_{\circ}$. Then for every $\nu=1,2, \ldots, 2 k$ there exists a half open Jordan path $\mathscr{P}_{\nu} \subset \mathfrak{L}$ which begins at $z_{\circ}$, contains $\ell_{\nu}$, and terminates on the boundary of $\Omega$. The latter means that $\overline{\mathscr{P}_{\nu}} \backslash \mathscr{P}_{\nu} \subset \partial \Omega$.

Proof. The construction of $\mathscr{P}_{\nu}$ begins with the extension of $\ell_{\nu}$ beyond its open endpoint along $\mathfrak{L}$ until it reaches a branching point of the dendrite, denote it by $z_{i_{1}} \in\left\{z_{1}, z_{2}, \ldots,\right\}$. Let us denote the extension by $\left[\gamma_{1}\right) \subset \mathfrak{L}$. This notation indicates that the arc $\left[\gamma_{1}\right)$ is half closed and half open. From its open endpoint at $z_{i_{1}}$ there emanate along $\mathfrak{L}$ a local half open arc the is different from $\left[\gamma_{1}\right)$. We prolong this arc until it reaches a branching point; say, $z_{i_{2}} \in\left\{z_{1}, z_{2}, \ldots,\right\}$. Denote this arc by $\left[\gamma_{2}\right) \subset \mathfrak{L}$. Of course the choice of $z_{i_{2}}$ is unique only if $z_{i_{1}}$ is a regular point of $u$. Continuing in this fashion we obtain a path

$$
\mathscr{P}_{\nu} \stackrel{\text { def }}{=}\left[\gamma_{1}\right) \cup\left[\gamma_{2}\right) \cup\left[\gamma_{3}\right) \cup \ldots
$$

This chain of arcs can be infinite, or can terminate with a half open arc $\left[\gamma_{N}\right) \subset \mathfrak{L}$. The latter happens if $\left[\gamma_{N}\right)$ has no open endpoint in $\Omega$. Since the dendrite $\mathfrak{L}$ contains no closed Jordan curve, we see that

(e) $\mathscr{P}_{\nu}$ does not intersect itself. In particular, the selected branching points $z_{i_{1}}, z_{i_{2}}, z_{i_{3}}, \ldots$ are distinct.

Let us parametrize $\mathscr{P}_{\nu}$ via a homeomorphism $\Psi_{\nu}:[0, \infty) \stackrel{\text { onto }}{\longrightarrow} \mathscr{P}_{\nu}$ so that

$$
\Psi_{\nu}(0)=z_{\circ}, \Psi_{\nu}(1)=z_{i_{1}}, \Psi_{\nu}(2)=z_{i_{2}}, \ldots, \Psi_{\nu}(N)=z_{i_{N}}, \ldots
$$

If the chain terminates, the parametrization of the last arc $\left[\gamma_{N}\right)$ is given by a homeomorphism $\Psi_{\nu}:[N, \infty) \stackrel{\text { onto }}{\longrightarrow}\left[\gamma_{N}\right)$.

(f) $\mathscr{P}_{\nu}$ converges to the boundary of $\Omega$, meaning that

$$
\lim _{t \rightarrow \infty} \operatorname{dist}\left\{\Psi_{\nu}(t), \partial \Omega\right\}=0
$$

Proof. Consider a locally finite cover of the dendrite by branching neighborhoods, $\mathfrak{L} \subset \mathfrak{B}_{z_{1}} \cup \mathfrak{B}_{z_{2}} \cup \ldots$. It follows the structure of $\mathfrak{L} \cap \mathfrak{B}_{z_{i}}$; that if $\mathscr{P}_{\nu}$ intersects $\mathfrak{B}_{z_{i}}$, it must contain $z_{i}$. On the other hand, $\Psi_{\nu}(t)$ cannot assume value $z_{i}$ twice; otherwise, we would have a closed Jordan curve in $\mathscr{P}_{\nu} \subset \mathfrak{L}$. In conclusion if $\mathscr{P}_{\nu}$ enters one of those branching neighborhoods it must leave 
it and never enter again it. Now, given any compact subset $G \Subset \Omega$ there is only a finite number of the branching neighborhoods in the family $\left\{\mathfrak{B}_{z_{1}}, \mathfrak{B}_{z_{2}}, \ldots\right\}$ which intersect $G$. Therefore, for $t$ sufficiently large $\Psi(t) \notin G$. This is exactly what (7.1) means.

(g) The End-Set of $\mathscr{P}_{\nu}$ is a continuum in $\partial \Omega$.

Proof. The term end-set of $\mathscr{P}_{\nu}$ refers to the cluster values of $\Psi_{\nu}(t)$ at $t=\infty$, in symbols

$$
\mathscr{P}_{\nu}\{\infty\} \stackrel{\text { def }}{=}\left\{\lim _{t_{j} \rightarrow \infty} \Psi_{\nu}\left(t_{j}\right) ; \text { whenever such a limit does exist }\right\}
$$

Equivalently,

$$
\mathscr{P}_{\nu}\{\infty\}=\overline{\mathscr{P}_{\nu}} \backslash \mathscr{P}_{\nu} \subset \partial \Omega
$$

The proof follows by looking at $\mathscr{P}_{\nu}\{\infty\}$ as intersection of a decreasing sequence of continua, namely $\mathscr{P}_{\nu}\{\infty\}=\bigcap_{\ell \geqslant 1} \overline{\Psi_{\nu}[\ell, \infty)}$.

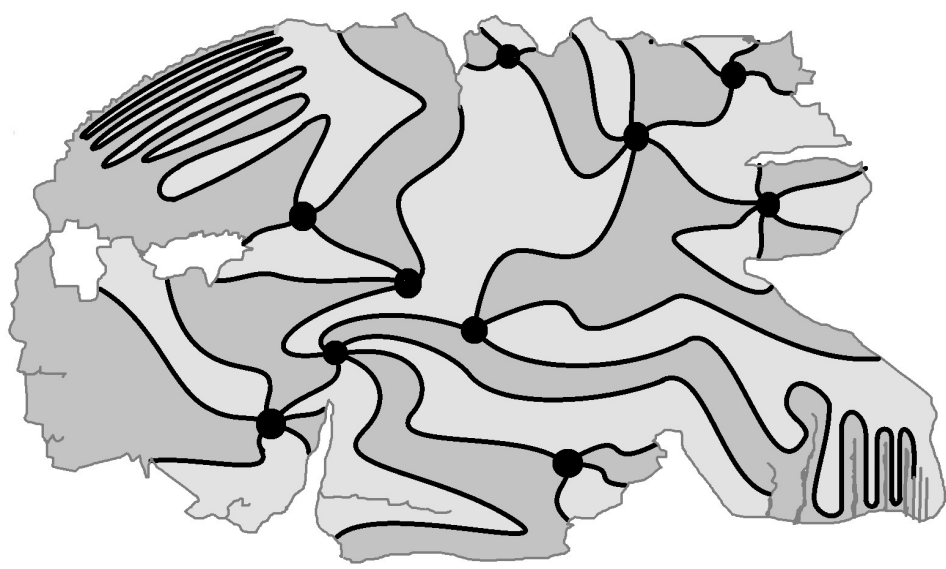

FiguRE 8. The shaded region marks a simply-connected domain $\Omega \subset \mathbb{R}^{2}$. As we see, $\Omega$ need not be the only complementary domain of $\partial \Omega$. Bullets are the critical points of $u$. The bold smooth open arcs are the level curves along which $\nabla u \neq 0$. These arcs together with the critical points form what we call $p$-harmonic dendrite. The dendrite is locally connected and contains no simple closed Jordan curve. Every path along the dendrite that emanates from a critical point approaches the boundary of $\Omega$, possibly in a bizarre way. 
Note that no pair $\mathscr{P}_{\nu}, \mathscr{P}_{\mu}, 1 \leqslant \nu, \mu \leqslant 2 k$ of different paths in $\mathfrak{L}$ emanating from $z_{\circ}$ can rejoin elsewhere in $\Omega$, since otherwise the dendrite would contain a closed Jordan curve. The union of such a pair

$$
\mathcal{C}=\mathcal{C}_{\mu}^{\nu} \stackrel{\text { def }}{=} \mathscr{P}_{\nu} \cup \mathscr{P}_{\mu}
$$

gives rise to what we call a generalized crosscut of $\Omega$ passing through $z_{\circ}$. It is convenient to parametrize $\mathcal{C}$ by a homeomorphism $\phi: \mathbb{R} \stackrel{\text { onto }}{\longrightarrow}$ $\mathcal{C}$ defined by the rule

$$
\phi(t)= \begin{cases}\Psi_{\nu}(t) & \text { for } t \geqslant 0 \\ \Psi_{\mu}(-t) & \text { for } t \leqslant 0\end{cases}
$$

Thus $\mathcal{C}$ has two end-sets (not necessarily disjoint or distinct). These are continua in $\partial \Omega$ defined by

$$
\mathcal{C}\{+\infty\}=\bigcap_{\ell \geqslant 0} \overline{\phi[\ell, \infty)} \text { and } \quad \mathcal{C}\{-\infty\}=\bigcap_{\ell \leqslant 0} \overline{\phi(-\infty, \ell]}
$$

\section{Generalized Crosscut of a Simply-Connected Domain}

Thus this section needs handling with great care. Indeed, intuitively obvious topological facts can be invalid for several reasons. Let $\Omega \subset \mathbb{R}^{2}$ be a domain. An open Jordan arc $\mathcal{C} \subset \Omega$ which has two different endpoints in $\partial \Omega$ is commonly called a crosscut of $\Omega$. It is well known in the complex function theory that every crosscut of a simply connected domain $\Omega \subset \mathbb{C}$ divides $\Omega$ into two simply connected domains, say $\Omega^{-}$and $\Omega^{+}$. That is, such a crosscut makes a disjoint decomposition $\Omega=\Omega^{-} \cup \mathcal{C} \cup \Omega^{+}$, where $\mathcal{C}=\partial \Omega^{-} \cap \partial \Omega^{+}$.

Let a bounded simply connected domain $\Omega \subset \mathbb{R}^{2}$ be fixed for the reminder of this section. Its boundary $\partial \Omega$ is a continuum which disconnects $\mathbb{R}^{2}$ into a number (possibly infinitely many) of connected open sets, called complementary domains of $\partial \Omega$. One of them is the domain $\Omega$ itself.

Definition 19. The term generalized crosscut of $\Omega$ refers to a set $\mathcal{C} \subset \Omega$ which is homeomorphic to the real line $\mathbb{R}$ and is closed in $\Omega$ with respect to the relative topology of $\Omega \subset \mathbb{R}^{2}$. Thus the set $\overline{\mathcal{C}} \backslash \mathcal{C}$, which we call the closure increment of $\mathcal{C}$, lies in $\partial \Omega$. Hereafter the notation $\bar{A}$ stands for the closure of a subset $A \subset \mathbb{R}^{2}$ in the usual topology of $\mathbb{R}^{2}$.

Theorem 20 (Generalized Crosscut). A generalized crosscut $\mathcal{C} \subset \Omega$ divides $\Omega$ into two simply-connected domains, denoted by $\Omega^{+}$and $\Omega^{-}$, 
We call them \pm components of $\Omega \backslash \mathcal{C}$. Thus we have a disjoint decomposition

$$
\Omega=\Omega^{-} \cup \mathcal{C} \cup \Omega^{+}
$$

The following inclusion holds:

(i)

$$
\mathcal{C} \subset \partial \Omega^{-} \cap \partial \Omega^{+}
$$

This inclusion is generally not an equality.

(ii) The boundary of $\Omega$ can be expressed by the formula,

$$
\partial \Omega=\left(\partial \Omega^{-} \backslash \mathcal{C}\right) \cup\left(\partial \Omega^{+} \backslash \mathcal{C}\right)
$$

which together with $(i)$ yields

(iii)

$$
\partial \Omega \subset \partial \Omega^{-} \cup \partial \Omega^{+}=\mathcal{C} \cup \partial \Omega
$$

Warning. It is possible that $\partial \Omega^{-}=\partial \Omega^{+}$. Construction of such a simply connected domain (with a nice crosscut) follows closely the familiar example of 3-lakes in $\mathbb{R}^{2}$ that share the same boundary, see Figure 10.

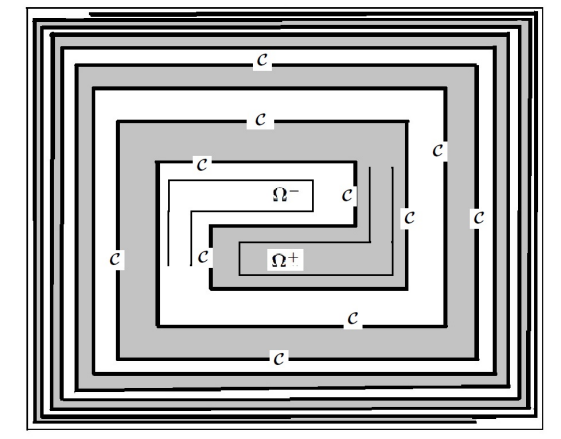

FiguRE 9. Generalized crosscut of a nice Jordan domain (rectangle) can be complicated. Here we have $\partial \Omega \subset \partial \Omega^{-}=\partial \Omega^{+}$.

Proof. A homeomorphism (say a conformal mapping) of the unit disk onto $\Omega$ takes concentric disks into an increasing family of smooth Jordan domains; say, 


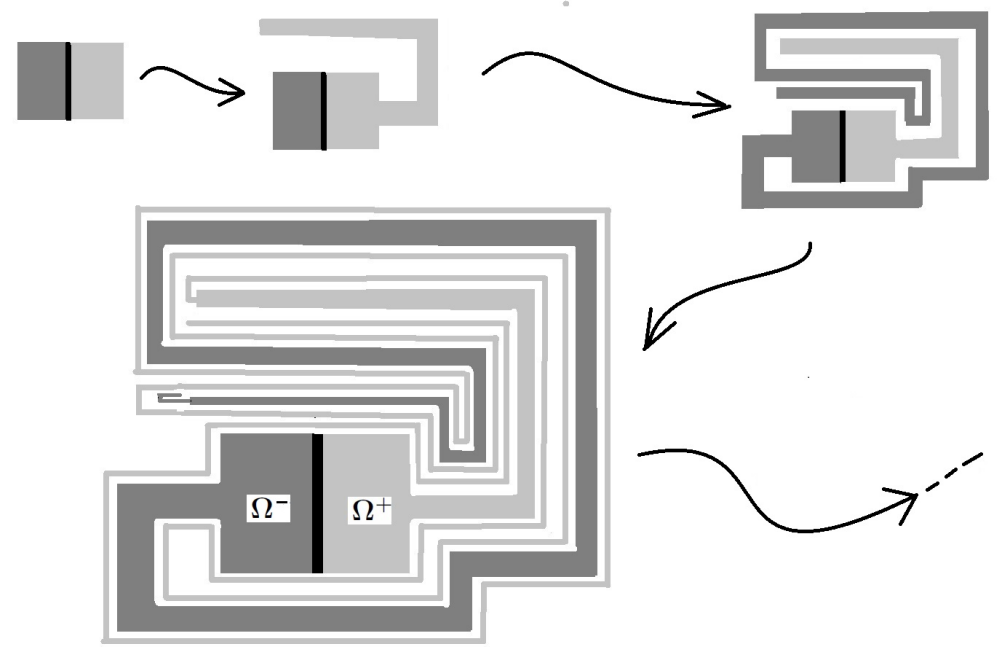

Figure 10. A nice straight line crosscut $\mathcal{C}$ of a simply connected domain, $\partial \Omega^{-}=\partial \Omega^{+}=\mathcal{C} \cup \partial \Omega$.

$$
\Omega_{0} \Subset \Omega_{1} \Subset \ldots \Subset \Omega_{n} \Subset \ldots \Subset \Omega, \bigcup_{n \geqslant 0} \Omega_{n}=\Omega, \mathcal{C} \cap \Omega_{0} \neq \varnothing
$$

Let us parametrize the general crosscut by a homeomorphism $\phi$ : $\mathbb{R} \stackrel{\text { into }}{\longrightarrow} \Omega$. That is,

$$
\mathcal{C}=\left\{z \in \Omega ; z=\phi(t),-\infty<t<+\infty, \phi(0) \stackrel{\text { def }}{=} z_{\circ} \in \Omega_{0}\right\}
$$

We have,

$$
\lim _{t \rightarrow-\infty} \operatorname{dist}\{\phi(t), \partial \Omega\}=0 \text { and } \lim _{t \rightarrow+\infty} \operatorname{dist}\{\phi(t), \partial \Omega\}=0
$$

As indicated before, the end-sets of $\mathcal{C}$, defined by the rules:

$$
\begin{aligned}
& \mathcal{C}\{+\infty\} \stackrel{\text { def }}{=}\left\{\text { all limits } \lim _{t_{n} \nearrow+\infty} \phi\left(t_{n}\right)\right\}=\bigcap_{\ell>0} \overline{\phi(\ell,+\infty)} \subset \partial \Omega \\
& \mathcal{C}\{-\infty\} \stackrel{\text { def }}{=}\left\{\text { all limits } \lim _{t_{n} \searrow-\infty} \phi\left(t_{n}\right)\right\}=\bigcap_{\ell<0} \overline{\phi(-\infty, \ell)} \subset \partial \Omega
\end{aligned}
$$

are continua in $\partial \Omega$. They need not be disjoint however. Denote the union of these end-sets by

$$
\mathcal{C}\{ \pm \infty\}=\mathcal{C}\{+\infty\} \cup \mathcal{C}\{-\infty\}=\overline{\mathcal{C}} \backslash \mathcal{C} \subset \partial \Omega
$$

Let $t_{n}^{+}>0$ be the largest parameter for which $\phi\left[0, t_{n}^{+}\right) \subset \Omega_{n}$ and $t_{n}^{-}<0$ the smallest parameter for which $\phi\left(t_{n}^{-}, 0\right] \subset \Omega_{n}$. The open 
Jordan arc $\mathcal{C}_{n} \stackrel{\text { def }}{=} \phi\left(t_{n}^{-}, t_{n}^{+}\right)$is a crosscut of the Jordan domain $\Omega_{n}$, with endpoints at $\phi\left(t_{n}^{-}\right)$and $\phi\left(t_{n}^{+}\right)$. Thus $\mathcal{C}_{n}$ splits $\Omega_{n}$ into exactly two simply connected Jordan domains $\Omega_{n}^{-}$and $\Omega_{n}^{+}$. In particular, for every $n$ we have a disjoint decomposition

$$
\Omega_{n}=\Omega_{n}^{-} \cup \mathcal{C}_{n} \cup \Omega_{n}^{+} \text {and the equation } \mathcal{C}_{n} \subset \partial \Omega_{n}^{-} \cap \partial \Omega_{n}^{+} .
$$

Letting $n \nearrow \infty$, we observe that $\left\{\Omega_{n}^{-}\right\}_{n \geqslant 1},\left\{\mathcal{C}_{n}\right\}_{n \geqslant 1}$ and $\left\{\Omega_{n}^{+}\right\}_{n \geqslant 1}$ are increasing sequences of sets. Their unions, denoted by $\Omega^{-}, \mathcal{C}$ and $\Omega^{+}$, respectively, the required generalized crosscut decomposition $\Omega=\Omega^{-} \cup \mathcal{C} \cup \Omega^{+}$.

To see the inclusion (i) $\mathcal{C} \subset \partial \Omega^{-} \cap \partial \Omega^{+}$we argue as follows: $\mathcal{C}_{n} \subset$ $\partial \Omega_{n}^{+}=\overline{\Omega_{n}^{+}} \backslash \Omega_{n}^{+} \subset \overline{\Omega^{+}} \backslash \Omega_{n}^{+}$. On the other hand, for every fixed integer $k \geqslant 1$ and all varaible $n \geqslant k$, we have the inclusions $\mathcal{C}_{k} \subset \mathcal{C}_{n} \subset$ $\overline{\Omega^{+}} \backslash \Omega_{n}^{+}$. Thus $\mathcal{C}_{k} \subset \bigcap_{n \geqslant k}\left(\overline{\Omega^{+}} \backslash \Omega_{n}^{+}\right)=\overline{\Omega^{+}} \backslash \bigcup_{n \geqslant k} \Omega_{n}^{+}=\overline{\Omega^{+}} \backslash \Omega^{+}=\partial \Omega^{+}$. This yields $\mathcal{C} \subset \bigcup_{k \geqslant 1} \mathcal{C}_{k} \subset \partial \Omega^{+}$. Similarly, $\mathcal{C} \subset \partial \Omega^{-}$, which yields the inclusion (i) .

To see the equation (ii), we need to show two opposite inclusions. Let us begin with:

$$
\begin{aligned}
\partial \Omega & =\bar{\Omega} \backslash \Omega=\overline{\Omega^{-} \cup \mathcal{C} \cup \Omega^{+}} \backslash \Omega=\left(\overline{\Omega^{-}} \cup \overline{\mathcal{C}} \cup \overline{\Omega^{+}}\right) \backslash \Omega=\overline{\Omega^{-}} \cup \overline{\Omega^{+}} \backslash \Omega \\
& =\left(\overline{\Omega^{-}} \backslash \Omega\right) \cup\left(\overline{\Omega^{+}} \backslash \Omega\right) \subset\left(\overline{\Omega^{-}} \backslash\left[\Omega^{-} \cup \mathcal{C}\right]\right) \cup\left(\overline{\Omega^{+}} \backslash\left[\Omega^{+} \cup \mathcal{C}\right]\right) \\
& =\left(\left[\overline{\Omega^{-}} \backslash \Omega^{-}\right] \backslash \mathcal{C}\right) \cup\left(\left[\overline{\Omega^{+}} \backslash \Omega^{+}\right] \backslash \mathcal{C}\right)=\left(\partial \Omega^{-} \backslash \mathcal{C}\right) \cup\left(\partial \Omega^{+} \backslash \mathcal{C}\right)
\end{aligned}
$$

On the other hand, since $\Omega^{-}$and $\overline{\Omega^{+}}$are disjoint, we have

$$
\begin{gathered}
\partial \Omega^{+} \backslash \mathcal{C}=\left(\overline{\Omega^{+}} \backslash \Omega^{+}\right) \backslash \mathcal{C}=\overline{\Omega^{+}} \backslash\left(\mathcal{C} \cup \Omega^{+}\right)=\overline{\Omega^{+}} \backslash\left(\Omega^{-} \cup \mathcal{C} \cup \Omega^{+}\right) \\
=\overline{\Omega^{+}} \backslash \Omega \subset \bar{\Omega} \backslash \Omega=\partial \Omega
\end{gathered}
$$

Similarly, we argue for the inclusion $\partial \Omega^{-} \backslash \mathcal{C} \subset \partial \Omega$. This completes the proof of the equality at $(i i)$, and the proof of Theorem 20 .

Next, we proceed to a detailed analysis of the complementary domains of the continuum

$$
\mathcal{K} \stackrel{\text { def }}{=} \mathcal{C} \cup \partial \Omega=\overline{\mathcal{C}} \cup \partial \Omega .
$$

At this point we invoke the following topological concept,

Definition 21 (Janiszewski space). A locally connected continuum $\mathfrak{X}$ is called Janiszewski space if the following holds true.

Whenever the intersection $\mathcal{C}_{0} \cap \mathcal{C}_{1}$ of two continua $\mathcal{C}_{0}, \mathcal{C}_{1} \subset \mathfrak{X}$ is not connected, the union $\mathcal{C}_{0} \cup \mathcal{C}_{1}$ disconnects $\mathfrak{X}$. 
Theorem 22. [31] The sphere $\mathbb{S}^{2}$ is a Janiszewski space.

See [35, Theorem 2. p.506] and also [14].

By Theorem of Janiszewski our continuum $\mathcal{K}$ disconnects the plane. There can be infinitely many complementary domains of $\mathcal{K}$, but only two of them "touch" $\mathcal{C}$. This is understood to mean the following:

Lemma 23. The \pm components $\Omega^{+}$and $\Omega^{-}$of $\Omega \backslash \mathcal{C}$ are among the complementary domains of $\mathcal{K}=\mathcal{C} \cup \partial \Omega$. These are the only ones whose boundaries intersect $\mathcal{C}$. Actually, by virtue of item (i) in Theorem 20, their boundaries contain $\mathcal{C}$ entirely.

Proof. Choose and fix a complementary domain $\Delta$ of $\mathcal{C} \cup \partial \Omega$ whose boundary contains a point in $\mathcal{C}$. Thus $\Delta$ intersects every neighborhood of $\mathcal{C}$; in particular, it intersects the entire domain $\Omega=$ $\Omega^{-} \cup \mathcal{C} \cup \Omega^{+}$. Since $\Delta \cap \mathcal{C} \subset \Delta \cap \mathcal{K}=\varnothing$, we have two possibilities:

$$
\left(A_{+}\right) \quad \Delta \cap \Omega^{+} \neq \varnothing \quad \text { or/end } \quad\left(A_{-}\right) \quad \Delta \cap \Omega^{-} \neq \varnothing
$$

It will appear later that only one of them may occur; precisely, $\Delta$ will be equal to equal to $\Omega^{+}$or $\Omega^{-}$.

Case $(+)$. Since $\Omega^{+}$is connected and disjoint with $\mathcal{K}$, it must lie entirly in the complementary domain of $\mathcal{K}$, in symbols $\Omega^{+} \subset \Delta$.

We now claim that $\Omega^{+}=\Delta$. Suppose, to the contrary, that $\Omega^{+} \varsubsetneqq \Delta$. This yields $\Delta \cap \partial \Omega^{+} \neq \varnothing$, in contradiction with $\Delta \cap \partial \Omega^{+} \subset \Delta \cap \mathcal{K}=$ $\varnothing$. Thus $\Delta=\Omega^{+}$.

Case $\left(A_{-}\right)$. As before, we conclude that $\Delta=\Omega^{-}$.

\subsection{A refinement of Theorem 20 and Lemma 23.}

We shall need to consider complementary domains of a sub-continuum of $\mathcal{C} \cup \partial \Omega$; say, $\mathcal{T} \stackrel{\text { def }}{=} \mathcal{C} \cup \Gamma$, where $\Gamma$ is a continuum in $\partial \Omega$. We need to ensure that $\mathcal{T}$ is a continuum that disconnects $\mathbb{R}^{2}$. For this we employ Janiszewski's theorem. Assume that $\Gamma$ contains both end-sets of the generalized crosscut $\mathcal{C}$. In symbols,

$$
\overline{\mathcal{C}} \backslash \mathcal{C} \subset \Gamma \subset \partial \Omega
$$

The idea behind our use of $\Gamma$ is that one of the complementary domains of $\mathcal{C} \cup \Gamma$, say $\mathcal{U}$, is contained in $\Omega$. By virtue of Lemma 23 this idea works well for $\Gamma=\partial \Omega$, in which case we have two such complementary 
domains, $\mathcal{U}=\Omega^{-} \subset \Omega$ and $\mathcal{U}=\Omega^{+} \subset \Omega$. Observe the general rule:

The smaller continuum $\mathcal{T} \subset \mathcal{C} \cup \partial \Omega$ the fewer complementary domains.

Unfortunately, it often happens ( that no complementary domain of $\mathcal{T}$ is contained in $\Omega$. The point is that the complement $\Lambda \stackrel{\text { def }}{=} \partial \Omega \backslash \Gamma$ need not be connected. We resolve this problem by imposing an extra assumptions on $\Gamma$; namely,

Theorem 24. Consider a continuum $\Gamma \subset \partial \Omega$ which contains $\overline{\mathcal{C}} \backslash \mathcal{C}$, and its complement $\Lambda \stackrel{\text { def }}{=} \partial \Omega \backslash \Gamma$ is connected (possibly empty). Then the boundary of one of the \pm components of $\Omega \backslash \mathcal{C}$ lies in $\mathcal{C} \cup \Gamma$. In symbols,

$$
\partial \Omega^{+} \subset \mathcal{C} \cup \Gamma \quad \text { or } \quad \partial \Omega^{-} \subset \mathcal{C} \cup \Gamma
$$

Let us point out in advance that the above hypotheses will be fulfilled in the forthcoming application, see Section 9.

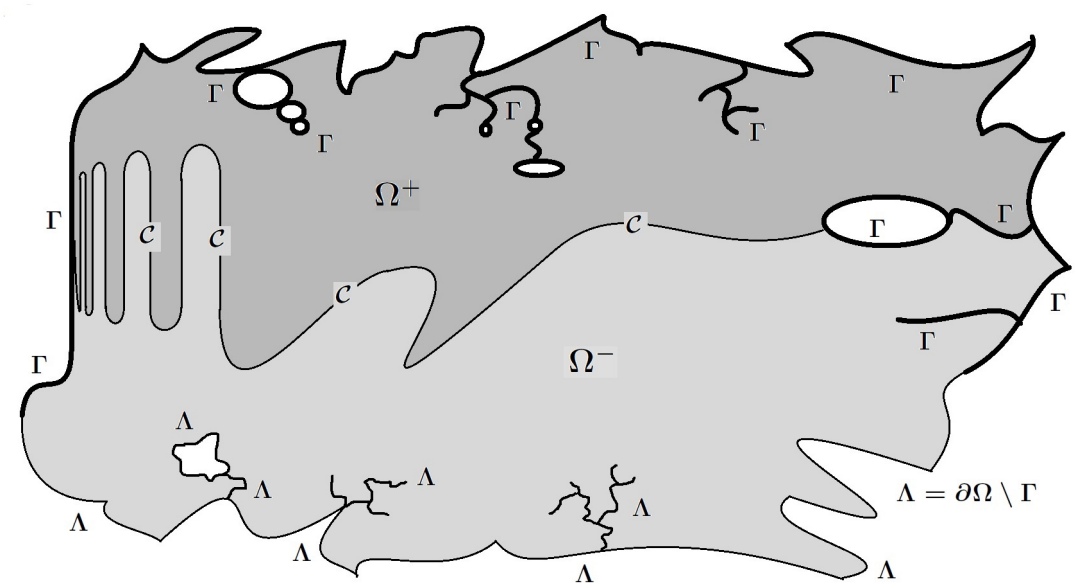

Figure 11. The complement $\Lambda=\partial \Omega \backslash \Gamma$ must be connected. Otherwise, no complementary domain of $\mathcal{C} \cup \Gamma$ lies in $\Omega$.

Proof. We have already seen that both inclusions in (8.3) hold if $\Gamma=$ $\partial \Omega$. Thus we assume that $\Lambda \neq \varnothing$. Striving for a contradiction, we also assume that

$$
\partial \Omega^{+} \not \subset \mathcal{C} \cup \Gamma \text { and } \partial \Omega^{-} \not \subset \mathcal{C} \cup \Gamma
$$


Equivalently,

$$
\Lambda \cap \partial \Omega^{+} \neq \varnothing \text { and } \Lambda \cap \partial \Omega^{-} \neq \varnothing
$$

The proof proceeds along the same lines as the proofs of Theorem 20 and Lemma 23, but the concluding details involve both connectedness of $\Lambda$ and connectedness of $\Gamma$.

Janiszewski's Theorem tells us that $\mathcal{C} \cup \Gamma$ disconnects $\mathbb{R}^{2}$ into (possibly infinitely many) complementary domains, usually fewer than those for $\mathcal{C} \cup \partial \Omega$. Among them there is at least one complementary domain which touches $\mathcal{C}$ (actually it contains $\mathcal{C}$ in its closure). Denote this complementary domain by $\Delta$. Thus, we have the following inclusion,

$$
\partial \Delta \subset \mathcal{C} \cup \Gamma
$$

To complete the proof of Theorem 24 it remains to show that:

Lemma 25. The following alternative holds:

$$
\text { either } \Delta=\Omega^{+} \text {or } \Delta=\Omega^{-}
$$

Proof. One of the \pm components $\Omega^{+}$or $\Omega^{-}$shares points with $\Delta$ near $\mathcal{C}$, say $\Omega^{+} \cap \Delta \neq \varnothing$. This yields

$$
\Omega^{+} \subset \Delta
$$

Indeed, $\Omega^{+}$is a connected subset of the complement of $\mathcal{C} \cup \partial \Omega$; hence is a connected subset of the complement of $\mathcal{C} \cup \Gamma$ as well. This means that $\Omega^{+}$lies entirely in one of the complementary domains of $\mathcal{C} \cup \Gamma$; obviously, the one which shares points with $\Omega^{+}$. The aim is to show that $\Delta=\Omega^{+}$. First observe that

$$
\Omega^{-} \cap \Delta=\varnothing
$$

Indeed, suppose to the contrary, that $\Omega^{-} \cap \Delta \neq \varnothing$. In much the same way as for the inclusion $\Omega^{+} \subset \Delta$, we infer that $\Omega^{-} \subset \Delta$. Thus,

$$
\Omega^{-} \cup \Omega^{+} \subset \Delta
$$

Choose and fix a point $p_{\circ} \in \mathcal{C} \subset \partial \Omega^{-} \cap \partial \Omega^{+}$. This point does not belong to $\Delta$, but there are points $p^{-} \in \Omega^{-}$and $p^{+} \in \Omega^{+}$arbitrarily close to $p_{\circ}$. We join them by a simple Jordan arc $\alpha \subset \Delta \cup\left\{p_{\circ}\right\}$ and by another simple Jordan arc $\beta \subset \Delta \backslash \alpha$. The union $\gamma=\alpha \cup \beta$ is a closed Jordan arc in $\Delta \cup\left\{p_{\circ}\right\}$ which disconnects $\mathbb{R}^{2}$ into two complementary domain; say, $\mathcal{D}^{-}$and $\mathcal{D}^{+}$. Thus $\mathbb{R}^{2}=\mathcal{D}^{-} \cup \gamma \cup \mathcal{D}^{+}$. The point $p_{\circ}$ divides the closed generalized crosscut $\overline{\mathcal{C}}$ into two connected subsets which lie in different complementary domains of $\gamma$, say

$$
\overline{\mathcal{C}} \backslash\left\{p_{\circ}\right\}=\overline{\mathcal{C}}_{+} \cup \overline{\mathcal{C}}_{-}, \quad \underset{32}{\text { where }} \overline{\mathcal{C}}_{+} \subset \mathcal{D}^{+} \text {and } \quad \overline{\mathcal{C}}_{-} \subset \mathcal{D}^{-}
$$


To justify the last two inclusions we observe that both connected sets $\overline{\mathcal{C}}_{+}$and $\overline{\mathcal{C}}_{-}$are disjoint with $\gamma$; one of them contains a point in $\mathcal{D}^{+}$ (near $p_{\circ}$ ) and the other contains a point in $\mathcal{D}^{-}$(near $p_{\circ}$ ).

The above two inclusions show that the end-sets $\mathcal{C}\{+\infty\}$ and $\mathcal{C}\{-\infty\}$, see the definition in (8.2), lie in different complementary domains of $\gamma$. Hence the continuum $\Gamma$, being a connection between these end-sets, must intersect $\gamma$. On the other hand $\gamma \subset \Delta \cup\left\{p_{\circ}\right\}$. The latter set is disjoint with $\Gamma$ because $\Delta$ is a complementary domain of $\mathcal{C} \cup \Gamma$. This is a clear a contradiction which proves (8.8).

We are now in a position to show that equality takes place in (8.7)

Suppose to the contrary, that $\Delta \supsetneq \Omega^{+}$. Thus, there are points $a \in \Delta \backslash \Omega^{+}$and $b \in \Delta \cap \Omega^{+}(b$ is near $\mathcal{C})$. We connect $a$ and $b$ by a Jordan arc $\tau \subset \Delta$. It must intersect $\partial \Omega^{+}$at some point $c \in \tau \cap \partial \Omega^{+} \subset \Delta \cap \partial \Omega$. This point cannot lie in $\Gamma \subset \partial \Omega$, because no point in $\Delta$ (a complementary domain of $\mathcal{C} \cup \Gamma$ ) lies in $\Gamma$. Thus $c \in \partial \Omega \backslash \Gamma=\Lambda$. On the other hand, in view of (8.4), there is also a point $d \in \Lambda \cap \partial \Omega^{-} \subset \mathbb{R}^{2} \backslash \Delta$, because of (8.8). We summarize by saying that the connected set $\Lambda \subset \partial \Omega$ contains both a point $c \in \Delta$ and a point $d \notin \Delta$. This means that there is a point in the intersection $\Lambda \cap \partial \Delta \subset \mathcal{C} \cup \Gamma$. But neither $\mathcal{C}$ nor $\Gamma$ contains a point in $\Lambda$. The above contradiction proves Lemma 25 .

This also completes the proof of Theorem 24.

\section{No CRITICAL POINTS OF $U$ AND $V$}

In this section we make the following standing assumptions on a $p$-harmonic map $H=U+i V$.

Definition 26. We assume that:

- $H \in \mathscr{C}(\bar{\Omega}, \mathbb{C})$ is a complex-valued continuous map defined on the closure of a bounded simply-connected domain $\Omega \subset \mathbb{C}$.

- Its real part $U$ and the imaginary part $V$ are $p$-harmonic functions in $\Omega$.

- The boundary map $H: \partial \Omega \stackrel{\text { onto }}{\longrightarrow} \partial \mathcal{Q}$ is monotone, where $\mathcal{Q}$ is a bounded convex domain in $\mathbb{C}$.

Proposition 27. If $H$ is as in the above definition, then both gradients $\nabla U$ and $\nabla V$ do not vanish in $\Omega$.

Proof. Suppose that, contrary to our claim, $\nabla U\left(z_{\circ}\right)=0$ at some point $z_{\circ} \in \Omega$; the case when $\nabla V$ vanishes is similar. Without loss of generality we assume that $U\left(z_{\circ}\right)=0$. From $z_{\circ}$ there emanate $2 k \geqslant 4$ 
of level paths $\mathscr{P}_{1}, \mathscr{P}_{2}, \ldots, \mathscr{P}_{2 k}$ converging to the boundary of $\Omega$. Their end-sets $\mathscr{P}_{1}\{+\infty\}, \mathscr{P}_{2}\{+\infty\}, \ldots, \mathscr{P}_{2 k}\{+\infty\}$, are continua in $\partial \Omega$, not necessarily disjoint. These end-sets are mapped via $H$ into $(U, V)$-plane; precisely, into the intersection of $\partial\lrcorner 0 Q$, with the vertical axis $(0, V)$. Since $\mathcal{Q}$ is convex, there are at most two components of such intersection, say $A$ and/or $B$. More specifically, if the axis cuts $\mathcal{Q}$ then it cuts $\partial \mathcal{Q}$ at exactly two points. These two points constitute the component $A$ and the component $B$, respectively. If, on the other hand, the axis only touches "tangentially" $\partial \mathcal{Q}$ along straight segment (possibly one point), then $A$ is the set of tangency and $B$ is void. Since $H: \partial \Omega \stackrel{\text { onto }}{\longrightarrow} \partial \mathcal{Q}$ is monotone, the preimage of each component is a continuum in $\partial \Omega$ which either contain or is disjoint with a given endset. But we have at most two such preimages $H^{-1}(A)$ and/or $H^{-1}(B)$. They contain all the end-sets $\mathscr{P}_{1}\{+\infty\}, \mathscr{P}_{2}\{+\infty\}, \ldots, \mathscr{P}_{2 k}\{+\infty\}$. Therefore, one of the preimages $H^{-1}(A)$ or $H^{-1}(B)$ must contains two end-sets, say $\mathscr{P}_{\nu}\{+\infty\}, \mathscr{P}_{\mu}\{+\infty\} \subset H^{-1}(A)$. We are going to apply Theorem 24 to the continuum $\Gamma \stackrel{\text { def }}{=} H^{-1}(A) \subset \partial \Omega$ and the generalized crosscut $\mathcal{C} \stackrel{\text { def }}{=} \mathscr{P}_{\nu} \cup \mathscr{P}_{\mu} \subset \Omega$. To justify our application we notice that not only $\Gamma$ but also its complement $\Lambda \stackrel{\text { def }}{=} \partial \Omega \backslash \Gamma$ is connected. This is true because $\Lambda$ is a pre-image of a connected subset of the boundary of $\mathcal{Q}$, namely $\Lambda=H^{-1}(\partial \mathcal{Q} \backslash A)$, see Definition 3 .

Now Theorem 24 tells us that there is an open subset $\mathcal{O} \subset \Omega$; namely, one of the \pm components of $\Omega \backslash \mathcal{C}$, such that

$$
\partial \mathcal{O} \subset \mathcal{C} \cup \Gamma \text {. Thus } U \text { vanishes on } \partial \mathcal{O} \text {, }
$$

By $\max / \min$ principle and unique continuation property of $p$-harmonic functions, we conclude that $U \equiv 0$ in $\mathcal{O}$ and hence $U \equiv 0$ on $\Omega$. This is a clear contradiction of the fact that $H: \partial \Omega \stackrel{\text { onto }}{\longrightarrow} \partial \mathcal{Q}$, completing the proof of Proposition 27.

9.1. Jacobian determinant. The next step is to examine the Jacobian determinant $J_{H}(z)=U_{x} V_{y}-U_{y} V_{x}, z=x+i y$. Our standing assumptions are formulated in Definition 26. The goal is to show that $J_{H}(z) \neq 0$ in $\Omega$. In the contrary case we would have a point $z_{\circ} \in \Omega$ at which the gradients $\nabla U\left(z_{\circ}\right)$ and $\nabla U\left(z_{\circ}\right)$ are linearly dependent; say, for some real coefficients

$$
\alpha \nabla U\left(z_{\circ}\right)+\beta \nabla V\left(z_{\circ}\right)=0, \quad \alpha^{2}+\beta^{2}=1, \alpha \neq 0 \neq \beta
$$

For the latter condition, we recall that Proposition 27 rules out the case $\alpha=0$ and the case $\beta=0$. 
Remark 28. The case of harmonic mappings $(p=2)$ follows from Proposition 27 by linear change of variables in the $(U, V)$-plane. Indeed, the mapping $\widetilde{H}=\widetilde{U}+i \widetilde{V}$, defined by the rotation $\widetilde{H}=$ $\left[\begin{array}{cc}\alpha & \beta \\ -\beta & \alpha\end{array}\right] H$, is again harmonic and takes $\Omega$ onto a convex region $\widetilde{\Omega}=$ $\left[\begin{array}{cc}\alpha & \beta \\ -\beta & \alpha\end{array}\right] \Omega$. Therefore, its coordinate function

$$
W(z) \stackrel{\text { def }}{=} \alpha U(z)+\beta V(z)
$$

has no critical points in $\Omega$, as desired.

Such nice direct argument, first presented in the original paper by Kneser [34], does not apply to nonlinear PDEs. Nevertheless, once we establish an elliptic PDE for $W$ (by averaging a family of $p$-harmonic equations) the proof will continue in the same way.

\section{An Elliptic Equation for $W=\alpha U+\beta V$}

The derivation of a linear elliptic equation for $W$ is based upon an idea found in [7]. However, our computation differs from that in [7] in a number of details.

Recall the $p$-harmonic map $H=U+i V$ with $\nabla U(z) \neq 0$ and $\nabla V(z) \neq 0$, so both $U$ and $V$ are $\mathscr{C}^{\infty}$-smooth. Then

Proposition 29. To every linear combination $W \stackrel{\text { def }}{=} \alpha U+\beta V$, with real coefficients, $\alpha^{2}+\beta^{2}=1$, there corresponds a symmetric matrix field $\mathcal{A}=\mathcal{A}(z)$ defined everywhere in $\Omega$ such that

$$
\mathcal{A}(z) \nabla W=|\alpha \nabla U|^{p-2} \alpha \nabla U+|\beta \nabla V|^{p-2} \beta \nabla V
$$

everywhere in $\Omega$. The matrix $\mathcal{A}(z) \in \mathbb{R}_{\mathrm{sym}}^{2 \times 2}$ satisfies the ellipticity bounds,

$$
\lambda_{p}|\xi|^{2} \leqslant \frac{\langle\mathcal{A}(z) \xi \mid \xi\rangle}{(|\alpha \nabla U(z)+| \beta \nabla V(z) \mid)^{p-2}} \leqslant \Lambda_{p}|\xi|^{2}
$$

for all vectors $\xi \in \mathbb{R}^{2}$ and every point $z \in \Omega$.

Some explicit bounds of the constants $\lambda_{p}$ and $\Lambda_{p}$ are given in (10.11).

Corollary 30. We observe that each term in the right hand side of (10.1) is a $\mathscr{C}^{\infty}$-smooth divergence free vector field, because $U$ and $V$ are $p$-harmonic. Therefore, we have an elliptic equation

$$
\operatorname{div} \mathcal{A}(z) \nabla W=0
$$

Remark 31. It will be shown that $\mathcal{A}$ is $\mathscr{C}^{\infty}$-smooth near every critical point of $W$. Then we shall deduce that in fact $W$ has no critical points, see the conclusion of Section 11. 
Proof of Equation (10.1). Consider a one-parameter family of $\mathscr{C}^{\infty}$-smooth functions

$W_{t} \stackrel{\text { def }}{=} t \alpha U-(1-t) \beta V=t W-\beta V=(t-1) W+\alpha U, \quad 0 \leqslant t \leqslant 1$

and their gradient

$$
\begin{aligned}
\nabla W_{t} & =t \alpha \nabla U-(1-t) \beta \nabla V=-\beta \nabla V+t \nabla W \\
& =\alpha \nabla U-(1-t) \nabla W
\end{aligned}
$$

Hence, for every $0 \leqslant t \leqslant 1$, by triangle inequalities,

$$
2\left|\nabla W_{t}\right| \geqslant|\beta \nabla V|-t|\nabla W|+|\alpha \nabla U|-(1-t)|\nabla W|
$$

$$
=|\alpha \nabla U|+|\beta \nabla V|-|\nabla W|
$$

We see that $\nabla W_{t}(z) \neq 0$ whenever

$$
|\nabla W(z)|<|\alpha||\nabla U(z)|+|\beta||\nabla V(z)|
$$

In particular, near every critical point of $W$ we have $\nabla W_{t}(z) \neq 0$ for every $t \in[0,1]$. In the region satisfying the above inequality we define the coefficient matrix of (10.3) by,

$$
\mathcal{A}(z) \stackrel{\text { def }}{=} \int_{0}^{1}\left|\nabla W_{t}(z)\right|^{p-2}\left(\mathbf{I}+(p-2) \frac{\nabla W_{t}(z) \otimes \nabla W_{t}(z)}{\left|\nabla W_{t}(z)\right|^{2}}\right) \mathrm{d} t
$$

Obviously $\mathcal{A}$ is $\mathscr{C}^{\infty}$-smooth in the above region. Now consider the complementary set in which

$$
|\nabla W(z)| \geqslant|\alpha||\nabla U(z)|+|\beta||\nabla V(z)|
$$

For some points in this set, we still may have $\nabla W_{t}(z) \neq 0$ for all $t \in$ $[0,1]$. In this case the matrix $\mathcal{A}(z)$ will be defined by the same formula (10.5). There remains the case when $\nabla W(z) \neq 0$ but $\nabla W_{t_{\circ}}(z)=0$ for some parameter $t_{\circ} \in[0,1]$. If so, we notice that there can be at most one such parameter $t_{\circ}$; namely,

$$
-\beta \nabla V(z)+t_{\circ} \nabla W(z)=0=\alpha \nabla U(z)-\left(1-t_{\circ}\right) \nabla W(z)
$$

Thus

$$
t_{\circ}=|\beta| \frac{|\nabla V(z)|}{|\nabla W(z)|} \quad \text { and } \quad 1-t_{\circ}=|\alpha| \frac{|\nabla U(z)|}{|\nabla W(z)|}
$$

Furthermore, for every $t \in[0,1]$ the gradients $\nabla W_{t}(z)$ are parallel to $\nabla W(z)$; that is, $\nabla W_{t}(z)=\left(t-t_{\circ}\right) \nabla W(z)$. Now the integrand at 
(10.5 is well defined, except for $t=t_{\circ}$. Therefore, following (10.5), we put

$$
\mathcal{A}(z) \stackrel{\text { def }}{=}|\nabla W(z)|^{p-2}\left(\mathbf{I}+(p-2) \frac{\nabla W(z) \otimes \nabla W(z)}{|\nabla W(z)|^{2}}\right) \int_{0}^{1}\left|t-t_{\circ}\right|^{p-2} \mathrm{~d} t
$$

The latter integral can explicitly be computed in terms of $z$,

$$
\int_{0}^{1}\left|t-t_{\circ}\right|^{p-2} \mathrm{~d} t=\frac{t_{\mathrm{o}}^{p-1}+\left(1-t_{\circ}\right)^{p-1}}{p-1}=\frac{|\beta \nabla V(z)|^{p-1}+|\alpha \nabla U(z)|^{p-1}}{(p-1)|\nabla W(z)|^{p-1}}
$$

and estimated from below and from above

$$
\frac{1}{p-1} \min \left\{1,2^{2-p}\right\} \leqslant \frac{t_{\circ}^{p-1}+\left(1-t_{\circ}\right)^{p-1}}{p-1} \leqslant \frac{1}{p-1} \max \left\{1,2^{2-p}\right\}
$$

Having defined $\mathcal{A}=\mathcal{A}(z)$ we now proceed to the proof of the identity (10.1).

Case 1. $\nabla W(z)=0$. The equation (10.1) holds because the left and the right hand side vanish, $\alpha \nabla U(z)=-\beta \nabla V(z)$.

Case 2. $\nabla W(z) \neq 0$ but $\nabla W_{t_{\circ}}(z)=0$ for some parameter $t_{\circ} \in[0,1]$. We use formula (10.7). Accordingly,

$$
\begin{aligned}
\mathcal{A}(z) \nabla W= & |\nabla W|^{p-2}(\nabla W+(p-2) \nabla W) \int_{0}^{1}\left|t-t_{\circ}\right|^{p-2} \mathrm{~d} t \\
& =\left(t_{\circ}^{p-1}+\left(1-t_{\circ}\right)^{p-1}\right)|\nabla W|^{p-2} \nabla W \\
& =\left|t_{\circ} \nabla W\right|^{p-2} t_{\circ} \nabla W+\left|\left(1-t_{\circ}\right) \nabla W\right|^{p-2}\left(1-t_{\circ}\right) \nabla W \\
& =|\beta \nabla V|^{p-2} \beta \nabla V+|\alpha \nabla U|^{p-2} \alpha \nabla U, \quad \text { by (10.6). }
\end{aligned}
$$

Case 3. $\nabla W(z) \neq 0$ and $\nabla W_{t}(z) \neq 0$ for all parameters $t \in[0,1]$. In this case formula (10.5) applies. Observe that $\frac{\mathrm{d}}{\mathrm{d} t} \nabla W_{t}=\nabla W$, so we have

$$
\begin{aligned}
& \frac{\mathrm{d}}{\mathrm{d} t}\left(\left|\nabla W_{t}\right|^{p-2} \nabla W_{t}\right) \\
= & \left|\nabla W_{t}\right|^{p-2} \nabla W+(p-2)\left|\nabla W_{t}\right|^{p-4}\left\langle\nabla W_{t} \mid \nabla W\right\rangle \nabla W_{t} \\
= & \left|\nabla W_{t}(z)\right|^{p-2}\left(\mathbf{I}+(p-2) \frac{\nabla W_{t}(z) \otimes \nabla W_{t}(z)}{\left|\nabla W_{t}(z)\right|^{2}}\right) \nabla W
\end{aligned}
$$

Integrating from $t=0$ to $t=1$ gives the identity

$$
\left|\nabla W_{1}\right|^{p-2} \nabla W_{1}-\left|\nabla W_{0}\right|^{p-2} \nabla W_{0}=\mathcal{A}(z) \nabla W,
$$

which is the same as (10.1). The proof is complete. 
Proof of (10.2). Although the explicit constants $\lambda_{p}$ and $\Lambda_{p}$ play no role in the sequel, some computation is worth carrying out. We are going to reduce (10.2) to an inequality which is well known in the study of the monotone operator $X \rightsquigarrow|X|^{p-2} X$. Namely, for vectors $X \neq Y$ in any inner product space, we have

$$
m_{p} \leqslant \frac{\left\langle|X|^{p-2} X-|Y|^{p-2} Y \mid X-Y\right\rangle}{(|X|+|Y|)^{p-2}|X-Y|^{2}} \leqslant M_{p}
$$

with certain constants $0<m_{p} \leqslant M_{p}<\infty$. Now consider a symmetric positive definite form in $\xi \in \mathbb{R}^{2}$, with a given nonzero vector $\mathbf{W} \in \mathbb{R}^{2}$,

$$
\begin{aligned}
& \left\langle\left(\mathbf{I}+(p-2) \frac{\mathbf{W} \otimes \mathbf{W}}{|\mathbf{W}|^{2}}\right) \xi \mid \xi\right\rangle \\
& =|\xi|^{2}+(p-2) \frac{\langle\mathbf{W} \mid \xi\rangle^{2}}{|\mathbf{W}|^{2}}=\left\{\begin{array}{l}
\leqslant \max \{1, p-1\}|\xi|^{2} \\
\geqslant \min \{1, p-1\}|\xi|^{2}
\end{array}\right.
\end{aligned}
$$

By virtue of (10.5) for every unit vector $\xi \in \mathbb{S}^{1}$ it holds that

$$
k_{p} \int_{0}^{1}\left|\nabla W_{t}(z)\right|^{p-2} \mathrm{~d} t \leqslant\langle\mathcal{A}(z) \xi \mid \xi\rangle \leqslant K_{p} \int_{0}^{1}\left|\nabla W_{t}(z)\right|^{p-2} \mathrm{~d} t
$$

where

$$
k_{p}=\min \{1, p-1\} \text { and } K_{p}=\max \{1, p-1\}
$$

We are left with the task of estimating (from below and from above) the integral in both sides of these estimates. Note that this integral, in case of the definition at (10.7), agrees with the expression $|\nabla W(z)|^{p-2} \int_{0}^{1}\left|t-t_{\circ}\right|^{p-2} \mathrm{~d} t$. For simplicity of notation, let us write $\nabla W_{t}(z) \stackrel{\text { def }}{=} Z_{t}=t X+(1-t) Y$, where $X=\alpha \nabla U$ and $Y=-\beta \nabla Y$. Therefore, $\frac{\mathrm{d}}{\mathrm{d} t} Z_{t}=X-Y$ and $Z_{1}=X, Z_{0}=Y$. We differentiate using product rule to obtain

$$
\begin{gathered}
\frac{\mathrm{d}}{\mathrm{d} t}\left\langle\left|Z_{t}\right|^{p-2} Z_{t} \mid X-Y\right\rangle=\left|Z_{t}\right|^{p-2}|X-Y|^{2}+(p-2)\left|Z_{t}\right|^{p-4}\left\langle Z_{t} \mid X-Y\right\rangle^{2} \\
\left\{\begin{array}{l}
\leqslant \max \{1, p-1\}\left|Z X_{t}\right|^{p-2}|X-Y|^{2} \\
\geqslant \min \{1, p-1\}\left|Z X_{t}\right|^{p-2}|X-Y|^{2}
\end{array}\right.
\end{gathered}
$$

Integrating from $t=0$ to $t=1$ we arrive at the inequalities

$$
\begin{aligned}
& \min \{1, p-1\} \int_{0}^{1}\left|Z X_{t}\right|^{p-2} \mathrm{~d} t \leqslant \\
& \frac{\left\langle|X|^{p-2} X-|Y|^{p-2} Y \mid X-Y\right\rangle}{|X-Y|^{2}}
\end{aligned}
$$




$$
\leqslant \max \{1, p-1\} \int_{0}^{1}\left|Z X_{t}\right|^{p-2} \mathrm{~d} t
$$

In view of (10.9) the expression in the middle of these inequalities is controlled from below and from above by $m_{p}(|X|+|Y|)^{p-2}$ and $M_{p}(|X|+$ $|Y|)^{p-2}$, respectively. We then find that

$$
\frac{m_{p}(|X|+|Y|)^{p-2}}{\max \{1, p-1\}} \leqslant \int_{0}^{1}|t X+(1-t) Y|^{p-2} \mathrm{~d} t \leqslant \frac{M_{p}(|X|+|Y|)^{p-2}}{\min \{1, p-1\}}
$$

This and (10.10) combined give the required estimate for all $\xi \in \mathbb{R}^{2}$ and $z \in \Omega$,

$$
\lambda_{p}|\xi|^{2} \leqslant \frac{\langle\mathcal{A}(z) \xi \mid \xi\rangle}{(|\alpha \nabla U(z)+| \beta \nabla V(z) \mid)^{p-2}} \leqslant \Lambda_{p}|\xi|^{2}
$$

where

$$
\lambda_{p} \stackrel{\text { def }}{=} \min \{p-1,1 / p-1\} m_{p} \leqslant \max \{p-1,1 / p-1\} M_{p} \stackrel{\text { def }}{=} \Lambda_{p}
$$

\section{The $\mathcal{A}$-Harmonic Dendrite of $W=\alpha U+\beta V$}

Let us now take a look at the level set $\mathfrak{L}=\mathfrak{L}_{W}=\{z \in \Omega ; W(z)=$ $0\}$. As one might expect the topological structure of $\mathfrak{L}_{W}$ is the same as that of the $p$-harmonic dendrite $\mathfrak{L}_{u}$. But the proof in case of $\mathfrak{L}_{W}$ is less involved. First note that $W \not \equiv$ constant, since otherwise the map $H=U+i V$ would take $\bar{\Omega}$ into a straight line segment in the $(U, V)$ plane, which is not the case. Concerning local structure of $\mathfrak{L}_{W}$, since $W$ is $\mathscr{C}^{\infty}$ smooth, through every regular point (where $\nabla W \neq 0$ ) there passes unique $\mathscr{C}^{\infty}$ - Jordan open arc. One cannot claim at this stage that the critical points of $W$ are isolated. That this is true will follow later from an analysis of the elliptic equation (10.3). It is vital that $\mathcal{A}$ is smooth near the critical points of $W$.

In $[16,34,48]$ harmonic functions are real and imaginary parts analytic functions. Analogous approach for $\mathcal{A}$-harmonic functions is provided via a Hodge star operator, see [9].

$*=\left[\begin{array}{cc}0 & -1 \\ 1 & 0\end{array}\right]: \mathbb{R}^{2} \stackrel{\text { onto }}{\longrightarrow} \mathbb{R}^{2} \quad$ (a counterclockwise rotation by 90 degrees)

In a simply connected domain every divergence free smooth vector field, such as $\mathcal{A}(z) \nabla W$, can be expressed (uniquely up to an additive constant) as $\mathcal{A}(z) \nabla W=-* \nabla \widetilde{W}$, whose $\widetilde{W} \in \mathscr{C}^{\infty}(\Omega)$ is called $\mathcal{A}$ conjugate function. Let $\mathcal{A}=\left[\begin{array}{cc}\mathcal{A}_{11} & \mathcal{A}_{12} \\ \mathcal{A}_{21} & \mathcal{A}_{22}\end{array}\right]$. 
Then the complex function $F=W+i \widetilde{W} \in \mathscr{C}^{\infty}(\Omega)$ solves a first order elliptic system ,

$$
\begin{gathered}
\frac{\partial F}{\partial \bar{z}}=\mu(z) \frac{\partial F}{\partial z}+\nu(z) \frac{\overline{\partial F}}{\partial z}, \quad|\mu(z)|+|\nu(z)| \leqslant k(z)<1 \\
\mu(z)=\frac{\mathcal{A}_{22}(z)-\mathcal{A}_{11}(z)-2 i \mathcal{A}_{12}(z)}{\operatorname{det}[\mathbf{I}+\mathcal{A}(z)]}, \quad \nu(z)=\frac{1-\operatorname{det} \mathcal{A}(z)}{\operatorname{det}[\mathbf{I}+\mathcal{A}(z)]}
\end{gathered}
$$

At the given point $z \in \Omega$ the ellipticity bound $k=k(z)$ can be determined from the estimates in (10.2). In particular, $k$ can be made continuous in $\Omega$ so the system (11.1) becomes uniformly elliptic on compact subdomains of $\Omega$ and the complex coefficients $\mu=\mu(z)$ and $\nu=\nu(z)$ are $\mathscr{C}^{\infty}$-smooth near the critical points of $W$. Consequently, our solution $F$ is quasiregular on every subdomain $\Omega^{\prime} \Subset \Omega$ and, as such, admits Stoilow's factorization,

$$
F(z)=\Phi(\chi(z))
$$

Here $\chi: \Omega^{\prime} \stackrel{\text { into }}{\longrightarrow} \mathbb{C}$ is a quasiconformal homeomorphism and $\Phi$ is holomorphic in $\chi\left(\Omega^{\prime}\right)$. Let us take advantage of this factorization and examine the behavior of $F$ near the critical point $z_{\circ} \in \Omega$ of part $W=\Re e F$. Clearly, $z_{\circ}$ is also a critical point of the $\mathcal{A}$-harmonic conjugate function $\widetilde{W}$, meaning that

$$
\frac{\partial F}{\partial z}\left(z_{\circ}\right)=\frac{\partial F}{\partial \bar{z}}\left(z_{\circ}\right)=0
$$

Remark 32. Caution should be exercised. Stoilow's factorization shows that upon the change of the independent variable, say $z=\chi^{-1}(\xi)$, the solution $W=W(z)=W\left(\chi^{-1}(\xi)\right)=\Re e \Phi(\xi)$ becomes a harmonic function in $\xi$. However, the corresponding point $\xi_{\circ}=\chi\left(z_{\circ}\right)$ is generally not a critical point of the harmonic function $\Re e \Phi$. Consider the example, $F(z)=|z|^{2} z, \chi^{-1}(\xi)=|\xi|^{-2 / 3} \xi$ and $\Phi(\xi)=\xi$ at $z_{\circ}=\xi_{\circ}=0$. The reason is that $\chi^{-1}$ fails to be smooth near $\xi_{0}$.

Lemma 33. In a neighborhood of a critical point $z_{\circ}$ of $W$, there emanate from $z_{0}$ half open Jordan arcs $\ell_{1}, \ell_{2}, \ldots, \ell_{2 k}, k \geqslant 2$, along which $W$ assumes a constant value. These arcs do not join again, and outside those arcs $W(z) \neq W\left(z_{\circ}\right)$.

Proof. To simplify the writing, take $z_{\circ}=0, F\left(z_{\circ}\right)=0, \chi\left(z_{\circ}\right)=0$, so $\Phi(0)=0$. We may also modify Stoilow's factorization near $z_{\circ}=0$ as to obtain $\Phi(\xi)=\xi^{k}$. Here $k \geqslant 1$ is the order of zero of $\Phi$. Thus the factorization near the origin takes the form.

$$
F(z)=[\chi(z)]^{k} \quad\left(|\chi(z)|_{40} \leqslant r, \text { with sufficiently small } r\right)
$$


Claim 1. We have $k \geqslant 2$.

Proof of Claim 1. Suppose that $k=1$, contrary to our claim. Thus $F$ is a quasiconformal homeomorphism. Its inverse, denoted by $G(\xi) \stackrel{\text { def }}{=}$ $\chi^{-1}(\xi)$, is also quasiconformal. Elementary chain rule, see [9, Equations (2.49), (2,50)] for details, leads to the following formulas

$$
\left.\frac{\partial F}{\partial \bar{z}}=-J(z, F) \frac{\partial G}{\partial \bar{\xi}}, \text { and } \frac{\partial F}{\partial z}=J(z, F)\right) \frac{\overline{\partial G}}{\partial \xi}, \quad z=G(\xi)
$$

Substituting to (11.1), we arrive at a quasilinear elliptic system for $G$,

$$
\frac{\partial G}{\partial \bar{\xi}}+\mu(G) \frac{\overline{\partial G}}{\partial \xi}+\nu(G) \frac{\partial G}{\partial \xi}=0
$$

The Schauder Regularity Theory tells us, see Theorem 15.0.7 in [9], that $G$ is $\mathscr{C}^{\infty}$ - smooth near the origin. Now we have the required contradiction since $\mathbf{I} \equiv D G(\xi) \cdot D F(z)$, where $D F\left(z_{\circ}\right)=\mathbf{0} \in \mathbb{R}^{2 \times 2}$. This proves Claim 1 .

We infer from the representation (11.2) that the level set $\{z ; W(z)=$ $\Re e F(z)=0\}$ is the pre-image under $\chi$ of the straight rays $L_{1}, L_{2}, \ldots, L_{2 k}$, where $L_{\nu}=\left[0, r e^{\frac{(2 \nu-1) \pi i}{2 k}}\right)$ for $\nu=1,2, \ldots, 2 k$. These are precisely the Jordan $\operatorname{arcs} \ell_{1}, \ell_{2}, \ldots, \ell_{2 k} \subset \mathfrak{L}, 1 \leqslant k<\infty$, in Lemma 33 .

Now the situation is in all respects similar to that of critical level set of $U$, which we discussed in Sections 9. The rest of the study of $\nabla W$ is essentially a repetition of the arguments used to proof that $\nabla U \neq 0$. The outcome is that $\nabla W \neq 0$.

Returning to the condition (9.1) we infer that the Jacobian of $H=$ $U+i V$ does not vanish in $\Omega$. Therefore, $H: \Omega \stackrel{\text { into }}{\longrightarrow} \mathbb{R}^{2}$ is a local diffeomorphism.

\section{Global Injectivity}

We begin with an elementary topological observation:

Lemma 34. Let $\mathbb{X}$ be a bounded domain in $\mathbb{R}^{2}$ and $f: \overline{\mathbb{X}} \rightarrow \mathbb{R}^{2}$ a continuous map such that

- $f(\mathbb{X})$ is open, thus a domain.

- $f(\partial \mathbb{X})=\partial \mathbb{Y}$, where $\mathbb{Y}$ is a Jordan domain

Then $f(\mathbb{X})=\mathbb{Y}$.

Proof. It simplifies the arguments, and causes no loss of generality, to assume that $\mathbb{Y}$ is the open unit disk. Just make a conformal transformation of $\mathbb{Y}$ onto the unit disk if necessary. We have the following inclusions: 
- $\partial f(\mathbb{X}) \subset \partial \mathbb{Y}$.

Indeed, $\partial f(\mathbb{X})=\overline{f(\mathbb{X})} \backslash f(\mathbb{X})=f(\overline{\mathbb{X}}) \backslash f(\mathbb{X})=f(\mathbb{X} \cup \partial \mathbb{X}) \backslash$ $f(\mathbb{X})=[f(\mathbb{X}) \cup f(\partial \mathbb{X})] \backslash f(\mathbb{X}) \subset f(\partial \mathbb{X})=\partial \mathbb{Y}$.

- $f(\mathbb{X}) \subset \mathbb{Y}$

To see this latter inclusion consider a point $y_{\circ} \in \overline{f(\mathbb{X})}$ that is furthest from the origin. This point certainly lies in $\partial f(\mathbb{X})$. The latter is a subset of $\partial \mathbb{Y}$, so $y_{0} \in \partial \mathbb{Y}$. Thus all other points in $\overline{f(\mathbb{X})}$ must lie in the closed unit disk $\overline{\mathbb{Y}}$. Consequently $f(\mathbb{X})$, being open, must lie in $\mathbb{Y}$.

- $\mathbb{Y} \subset f(\mathbb{X})$.

Suppose that, on the contrary, there is a point $y_{\circ} \in \mathbb{Y}$ which does not lie in $f(\mathbb{X})$. We connect $y_{\circ}$ with a point in $f(\mathbb{X})$ by a line segment. This segment intersects $\partial f(\mathbb{X})$ at some point inside the disk. This is a clear contradiction with $\partial f(\mathbb{X}) \subset \partial \mathbb{Y}$.

We now return to our map $H: \bar{\Omega} \rightarrow \mathbb{R}^{2}$ which takes $\partial \Omega$ onto the boundary of a convex domain $\mathcal{Q}$. Lemma 34 tells us that

$$
H(\Omega)=\mathcal{Q}
$$

In addition we know that $H$ is a local homeomorphism on $\Omega$. The general question arises when is a local homeomorphism a global homeomorphism? The early partial answers to this question can be traced back to the work of Banach and Mazur [10]. A very useful generalization of Banach-Mazur proposition has been published in 1975 by Ho in the following fashionable form.

Proposition 35. [23] Let $\mathbb{X}$ be pathwise connected and $\mathbb{Y}$ be simply connected Hausdorff spaces. A local homeomorphism $f: \mathbb{X} \rightarrow \mathbb{Y}$ is a global homeomorphism of $\mathbb{X}$ onto $\mathbb{Y}$ if and only if $f$ is proper.

What is left is to show that our map $H: \Omega \stackrel{\text { onto }}{\longrightarrow} \mathcal{Q}$ is proper. For this, we consider a compact set $F \Subset \Omega$ and its preimage $H^{-1}(F)$ under the map $H: \bar{\Omega} \stackrel{\text { onto }}{\longrightarrow} \overline{\mathcal{Q}}$. Certainly $H^{-1}(F)$ is a compact subset of $\bar{\Omega}$. This set cannot intersect $\partial \Omega$ because $H$ takes $\partial \Omega$ onto $\partial \mathcal{Q}$. Thus $H^{-1}(F)$ is a compact subset of $\Omega$, as desired.

Remark 36. Global injectivity of $H: \Omega \stackrel{\text { onto }}{\longrightarrow} \mathcal{Q}$ can also be deduced from a general theory of covering spaces. In fact, our local diffeomorphism $H: \Omega \stackrel{\text { onto }}{\longrightarrow} \mathcal{Q}$ is a covering map, because every fiber $H^{-1}\left(y_{\circ}\right) \subset$ $\Omega$, being discrete and compact, is a finite set. In particular, every point $y_{\circ} \in \mathcal{Q}$ has an evenly covered neighborhood via the projection map $H$. Now, since $\Omega$ is connected and $\mathcal{Q}$ is simply connected, the covering map $H: \Omega \stackrel{\text { onto }}{\longrightarrow} \mathcal{Q}$ is injective [47, Chapter IV] and [50]. 
The proof of THEOREM 4 is complete.

Acknowledgement. We acknowledge valuable discussions on topolical aspects of the subject with Professor Piotr Minc, Auburn University.

\section{REFERENCES}

1. G. Alessandrini, R. Magnanini, The index of isolated critical points and solutions of elliptic equations in the plane, Ann. Scuola Norm. Sup. Pisa Cl. Sci. (4) 19 (1992), no. 4, 567-589.

2. G. Alessandrini, R. Magnanini, Elliptic equations in divergence form, geometric critical points of solutions, and Stekloff eigenfunctions, SIAM J. Math. Anal. 25 (1994), no. 5, 1259-1268.

3. G. Alessandrini and V. Nesi, Univalent $\sigma$-harmonic mappings, Arch. Ration. Mech. Anal. 158 (2001), no. 2, 155-171.

4. G. Alessandrini and V. Nesi, Beltrami operators, non-symmetric elliptic equations and quantitative Jacobian bounds, Ann. Acad. Sci. Fenn. Math. 34 (2009), no. $1,47-67$.

5. G. Alessandrini and V. Nesi, Invertible harmonic mappings, beyond Kneser, Ann. Sc. Norm. Super. Pisa Cl. Sci. (5) 8 (2009), no. 3, 451-468.

6. G. Alessandrini and V. Nesi, Elliptic systems and material interpenetration, Funct. Approx. Comment. Math. 40 (2009), part 1, 105-115.

7. G. Alessandrini and M. Sigalotti, Geometric properties of solutions to the anisotropic p-Laplace equation in dimension two, Ann. Acad. Sci. Fenn. Math. 26 (2001), no. 1, 249-266.

8. G. Aronsson and P. Lindqvist, On p-harmonic functions in the plane and their stream functions, J. Differential Equations 74 (1988), no. 1, 157-178.

9. K. Astala, T. Iwaniec, and G. Martin, Elliptic partial differential equations and quasiconformal mappings in the plane, Princeton University Press, Princeton, NJ, 2009.

10. Banach, S., and Mazur, S. Über mehrdeutige stetige Abbildungen Studia Math. 5.1 (1934) 174-178.

11. P. Bauman, A. Marini and V. Nesi, Univalent solutions of an elliptic system of partial differential equations arising in homogenization, Indiana Univ. Math. J. 50 (2001), no. 2, 747-757.

12. P. Bauman and D. Phillips, Univalent minimizers of polyconvex functionals in two dimensions, Arch. Rational Mech. Anal. 126 (1994), no. 2, 161-181.

13. B. Bojarski and T. Iwaniec, p-harmonic equation and quasiregular mappings. Partial differential equations, Banach Center Publ., 19, PWN, Warsaw, (1987) $25-38$.

14. L. E. J. Brouwer, On the Structure of Perfect Sets of Points, Proc. Kon. Akad. van Wet. te Amsterdam 14 (1911), 137-147.

15. D. Bshouty and W. Hengartner, Univalent harmonic mappings in the plane, Handbook of complex analysis: geometric function theory. Vol. 2, 479-506, Elsevier, Amsterdam, (2005).

16. G. Choquet, Sur un type de transformation analytique généralisant la représentation conforme et définie au moyen de fonctions harmoniques, Bull. Sci. Math., 69, (1945), 156-165. 
17. P. Duren, Harmonic mappings in the plane, Cambridge University Press, Cambridge, (2004).

18. P. Duren and W. Hengartner, Harmonic mappings of multiply connected domains, Pacific J. Math. 180 (1997), no. 2, 201-220.

19. S. Gleason and T. Wolff, Lewy's harmonic gradient maps in higher dimensions, Comm. Partial Differential Equations 16 (1991), no. 12, 1925-1968.

20. D. H. Gottlieb, All the way with Gauss-Bonnet and the sociology of mathematics, Amer. Math. Monthly 103 (1996), no. 6, 457-469.

21. J. Heinonen, T. Kilpeläinen, and O. Martio, Nonlinear potential theory of degenerate elliptic equations, Oxford University Press, New York, 1993.

22. S. Hildebrandt and F. Sauvigny, Embeddedness and uniqueness of minimal surfaces solving a partially free boundary value problem, J. Reine Angew. Math. 422 (1991), 69-89.

23. Ho, C. W. A note on proper maps Proc. Amer. Math. Soc. 51 (1975), 237-241.

24. T. Iwaniec, A. Koski, and J. Onninen, Isotropic p-harmonic systems in $2 D$ Jacobian estimates and univalent solutions, Rev. Mat. Iberoam. 32 (2016), no. $1,57-77$.

25. T. Iwaniec, L. V. Kovalev, and J. Onninen, Diffeomorphic approximation of Sobolev homeomorphisms, Arch. Ration. Mech. Anal. 201 (2011), no. 3, 10471067.

26. T. Iwaniec and J. J. Manfredi, Regularity of p-harmonic functions on the plane, Rev. Mat. Iberoamericana 5 (1989), no. 1-2, 1-19.

27. T. Iwaniec and J. Onninen, Monotone Sobolev mappings of planar domains and surfaces Arch. Ration. Mech. Anal. 219 (2016), no. 1, 159-181.

28. T. Iwaniec and J. Onninen, Hyperelastic deformations of smallest total energy, Arch. Ration. Mech. Anal. 194 (2009), no. 3, 927-986.

29. T. Iwaniec and J. Onninen, Radó-Kneser-Choquet theorem, Bull. Lond. Math. Soc. 46 (2014), no. 6, 1283-1291.

30. T. Iwaniec and V. Šverák, On mappings with integrable dilatation, Proc. Amer. Math. Soc. 118 (1993), no. 1, 181-188.

31. S. Janiszewski, Sur les coupures du plan faites par des continus, Prace Mat.Fiz., 26 (1913), 11-63.

32. J. Jost, Univalency of harmonic mappings between surfaces, J. Reine Angew. Math. 324 (1981), 141-153.

33. D. Kalaj, Invertible harmonic mappings beyond the Kneser theorem and quasiconformal harmonic mappings, Studia Math. 207 (2011), no. 2, 117-136.

34. H. Kneser, Lösung der Aufgabe 41, Jahresber. Deutsch. Math.-Verein., 35, (1926), 123-124.

35. K. Kuratowski, Topology. Vol. II, Academic Press, New York-London; Państwowe Wydawnictwo Naukowe Polish Scientific Publishers, Warsaw (1968).

36. R. S. Laugesen, Injectivity can fail for higher-dimensional harmonic extensions. Complex Variables Theory Appl. 28 (1996), no. 4, 357-369.

37. H. Lewy, On the non-vanishing of the jacobian of a homeomorphism by harmonic gradients, Ann. of Math. (2) 881968 518-529.

38. A. Lyzzaik, Univalence criteria for harmonic mappings in multiply connected domains, J. London Math. Soc. (2) 58 (1998), no. 1, 163-171.

39. J. Malý, On increment of the tangent argument along a curve, Časopis Pěst. Mat. 106 (1981), no. 2, 186-196. 
40. J. Malý and W. P. Ziemer, Fine regularity of solutions of elliptic partial differential equations, Mathematical Surveys and Monographs, 51. American Mathematical Society, Providence, RI, (1997).

41. J. J. Manfredi, p-harmonic functions in the plane, Proc. Amer. Math. Soc. 103 (1988), no. 2, 473-479.

42. L.F. McAuley, Some fundamental theorems and problems related to monotone mappings In: Proceedings of First Conference on Monotone Mappings and Open Mappings. State Univ. of New York at Binghamton, N.Y., (1971).

43. A. Melas, An example of a harmonic map between Euclidean balls, Proc. Amer. Math. Soc. 117 (1993), no. 3, 857-859.

44. J. W. Milnor, On the total curvature of knots, Ann. of Math. (2) 52, (1950) 248-257.

45. C. B. Morrey, The Topology of (Path) Surfaces, Amer. J. Math. 57 (1935), no. $1,17-50$.

46. C.B. Morrey, On the solutions of quasi-linear elliptic partial differential quations, Trans. Amer. Math. Soc., 43 (1938), 126-166.

47. T. Parthasarathy, On global univalence theorems, Lecture Notes in Mathematics, 977. Springer-Verlag, Berlin-New York, (1983).

48. T. Radó, Aufgabe 41., Jahresber. Deutsch. Math.-Verein., 35, (1926), 49.

49. R. Schoen and S. T. Yau, On univalent harmonic maps between surfaces, Invent. Math. 44 (1978), no. 3, 265-278.

50. E. H. Spanier, Algebraic topology, Springer-Verlag, New York-Berlin, (1981).

Department of Mathematics, Syracuse University, Syracuse, NY 13244, USA

Email address: tiwaniec@syr.edu

Department of Mathematics, Syracuse University, Syracuse, NY 13244, USA and Department of Mathematics and Statistics, P.O.Box 35 (MaD) FI-40014 University of JyvÄskylä, Finland

Email address: jkonnine@syr.edu 